\title{
A CLOSED FORMULA FOR THE ASYMPTOTIC EXPANSION OF THE BERGMAN KERNEL
}

\author{
$\mathrm{HAO}$ XU
}

\begin{abstract}
We prove a graph theoretic closed formula for coefficients in the Tian-Yau-Zelditch asymptotic expansion of the Bergman kernel. The formula is expressed in terms of the characteristic polynomial of the directed graphs representing Weyl invariants. The proof relies on a combinatorial interpretation of a recursive formula due to M. Engliš and A. Loi.
\end{abstract}

\section{INTRODUCTION}

Let $M$ be a projective algebraic manifold in $\mathbb{C} P^{N}, N \geq \operatorname{dim}_{\mathbb{C}} M=n$. The hyperplane line bundle of $\mathbb{C} P^{N}$ restricts to an ample line bundle $L$ on $M$, which is called a polarization on $M$. A Kähler metric $g$ is called a polarized metric, if the corresponding Kähler form represents the first Chern class $c_{1}(L)$ of $L$ in $H^{2}(M, \mathbb{Z})$. Given any polarized Kähler metric $g$, there is a Hermitian metric $h_{L}$ on $L$ whose Ricci form is equal to

$$
\omega_{g}=\frac{\sqrt{-1}}{2 \pi} \sum_{i, j=1}^{n} g_{i \bar{j}} d z_{i} \wedge d z_{\bar{j}}
$$

Let $\mathcal{E}$ be a holomorphic vector bundle on $M$ of rank $r$ with a Hermitian metric $h_{\mathcal{E}}$. Then for any holomorphic sections $U_{1}, U_{2} \in H^{0}(M, \mathcal{E}(m))\left(\mathcal{E}(m):=\mathcal{E} \otimes L^{m}\right)$, we have the pointwise metric $\left\langle U_{1}(x), U_{2}(x)\right\rangle_{h_{\mathcal{E}} \otimes h_{L}^{m}}$ and the $L^{2}$-metric

$$
\left(U_{1}, U_{2}\right)=\int_{M}\left\langle U_{1}(x), U_{2}(x)\right\rangle_{h_{\mathcal{E}} \otimes h_{L}^{m}} \frac{\omega_{g}^{n}}{n !} .
$$

Let $S_{1}, \ldots, S_{d}$ be an orthonormal basis of $H^{0}\left(M, \mathcal{E} \otimes L^{m}\right)$ in the $L^{2}$-metric. The Bergman kernel is defined to be the following:

$$
B_{m}(x):=\sum_{j=1}^{d}\left\langle\cdot, S_{j}(x)\right\rangle S_{j}(x) \in \operatorname{End}\left(\mathcal{E} \otimes L^{m}\right) .
$$

Note that $B_{m}(x)$ is independent of the choice of orthonormal basis. In particular, when $\mathcal{E}=\mathbb{C}$,

$$
B_{m}(x)=\sum_{j=1}^{d}\left\|S_{j}(z)\right\|_{h_{L}^{m}}^{2} .
$$

The following asymptotic expansion was first proved by Zelditch [41] and Catlin [9, motivated by the convergence of Bergman metrics started in the paper of Tian [35] (cf. also [5, 31]) following a suggestion of Yau [40].

Theorem 1.1. (Zelditch, Catlin) With the notation above, there is an asymptotic expansion when $m \rightarrow \infty$ :

$$
B_{m}(x) \sim a_{0}(x) m^{n}+a_{1}(x) m^{n-1}+a_{2}(x) m^{n-2}+\cdots
$$

where $a_{k}(x) \in \operatorname{End}\left(\mathcal{E} \otimes L^{m}\right)$. More precisely,

$$
\left\|B_{m}(x)-\sum_{j=0}^{k} a_{j}(x) m^{n-j}\right\|_{C^{\mu}} \leq C_{k, \mu} m^{n-k-1},
$$

where $C_{k, \mu}$ depends on $k, \mu$ and the manifold $M$. 
In the case of $\mathcal{E}=\mathbb{C}, \mathrm{Lu}\left[23\right.$ computed $a_{k}(x)$ for $k \leq 3$ by using the peak section method 35] and proved that each $a_{k}(x)$ is a polynomial of the curvature and its covariant derivatives. The above theorem and Lu's computation of $a_{1}(x)$ have played a crucial role in Donaldson's breakthrough work 13. The theorem was generalized to symplectic manifolds for Boutet de Monvel-Guillemin's almost holomorphic sections by Shiffman and Zelditch [32], and Borthwick and Uribe [4. Dai, Liu and Ma 12. established the full off-diagonal expansion of the Bergman kernel on orbifolds and symplectic manifolds for the $\operatorname{Spin}^{c}$ Dirac operator by using the heat kernel method. See also [33, 29.

There are alternative derivations of these $a_{k}$ by Engliš [15] and Loi [22] using the Laplace integral, by Douglas and Klevtsov using path integral [14] when $\mathcal{E}=\mathbb{C}$. For general $\mathcal{E}$, X. Wang [37] computed $a_{1}$ for the first time; L. Wang [36] computed $a_{1}, a_{2}$; Ma and Marinescu [25, 27, 28] computed $a_{1}, a_{2}$ in the symplectic case and presented a recursive method to compute coefficients of the expansion of more general Bergman kernels as well as the kernel of Toeplitz operators; Berman, Berndtsson and Sjöstrand 2. gave a proof of Theorem 1.1 by microlocal analysis and showed a recursive algorithm for computing higher order terms; Liu and Lu 21] gave a proof of Theorem 1.1 using the complex geometric method and uncovered new geometric information about the expansion. The excellent monograph by Ma and Marinescu [26] contains a comprehensive introduction to the asymptotic expansion of the Bergman kernel and its applications.

In other contexts, the asymptotic expansion of the Bergman kernel also plays an important role in the Berezin quantization on Kähler manifolds [3]. We will discuss the Berezin transform briefly in Section 3 It was applied to define the Berezin $\star$-product on Kähler manifolds. Reshetikhin and Takhtajan [30] obtained a formula of the Berezin $\star$-product in terms of partition functions of Feynman graphs. More explicit graph-theoretic formulae of the Berezin and Berezin-Toeplitz $\star$-products were obtained in [38, 39]. We remark that Kontsevich's celebrated formula [20] for a $\star$-product on Poisson manifolds was also written as a summation over graphs.

In order to state the main results in this paper, we assume $\mathcal{E}=\mathbb{C}$ and introduce some terminologies in graph theory.

A digraph (directed graph) $G=(V, E)$ is defined to be a finite set $V$ (whose elements are called vertices) and a multiset $E$ of ordered pairs of vertices, called directed edges. Throughout the paper, we allow a digraph to have loops and multi-edges. The adjacency matrix $A=A(G)$ of a digraph $G$ with $n$ vertices is a square matrix of order $n$ whose entry $A_{i j}$ is the number of directed edges from vertex $i$ to vertex $j$. The outdegree $\operatorname{deg}^{+}(v)$ and indgree $\operatorname{deg}^{-}(v)$ of a vertex $v$ are defined to be the number of outward and inward edges at $v$ respectively.

A digraph $G$ is called connected if the underlying undirected graph is connected, and strongly connected if there is a directed path from each vertex in $G$ to every other vertex.

We call a directed graph $G=(V, E)$ stable if at each vertex $v$ both the outdegree and indegree are no less than 2. The set of stable graphs will be denoted by $\mathcal{G}$. The weight of $G$ is defined to be $|E|-|V|$. We will define a natural function $z(G)$ on $\mathcal{G}$ such that the coefficients $a_{k}$ can be written as a sum over $\mathcal{G}(k)$, the set of stable graphs of weight $k$ (see Section 5 for details)

$$
a_{k}(x)=\sum_{G \in \mathcal{G}(k)} z(G) \cdot G, \quad z(G) \in \mathbb{Q} .
$$

So we may regard $z$ as a map from the set $\mathcal{G}$ of all stable graphs to $\mathbb{Q}$, from which we can easily recover the curvature-tensor expression of these $a_{k}$ 's (see Example 6.11 where $a_{1}$ and $a_{2}$ are computed, and Appendix $\mathrm{A}$ where $a_{3}$ is computed).

The main result of this paper is the following theorem.

Theorem 1.2. Let $G=(V, E) \in \mathcal{G}$ be a stable graph with the adjacency matrix $A$.

i) If $G$ is a disjoint union of connected subgraphs $G=G_{1} \cup \cdots \cup G_{n}$, then we have

$$
z(G)=\prod_{j=1}^{n} z\left(G_{j}\right) /\left|\operatorname{Sym}\left(G_{1}, \ldots, G_{n}\right)\right|,
$$

where $\operatorname{Sym}\left(G_{1}, \ldots, G_{n}\right)$ denote the permutation group of these $n$ connected subgraphs. 
ii) If $G$ is connected but not strongly connected, then

$$
z(G)=0 \text {. }
$$

iii) If $G$ is strongly connected, then

$$
z(G)=-\frac{\operatorname{det}(A-I)}{|\operatorname{Aut}(G)|},
$$

where $I$ is the identity matrix.

We computed $z(G)$ for $G \in \mathcal{G}(k), k \leq 4$ using Loi's recursive formula (47). They match with values computed by the above theorem (see the appendix).

As pointed out by the referee, it would be interesting to see the implication of the above theorem on the relation between the Bergman kernel on Kähler manifolds and the path integral (cf. [14]). We hope our work will find application in Fefferman's program of studying the Bergman kernel of a strong pseudoconvex domain as an analogy of the heat kernel of Riemannian manifolds (cf. [1, 15, 17, 18, 38]).

We also obtained some interesting combinatorial identities, for example we proved the following formula for Bernoulli numbers $B_{k}$ in corollary 7.7

$$
B_{k}=(-1)^{k} k \sum_{G \in \mathcal{G}(k)} \frac{\operatorname{det}(A-I)}{|\operatorname{Aut}(G)|} \cdot \epsilon(G) \prod_{v \in V}\left(\operatorname{deg}^{+}(v)-1\right) !, \quad k \geq 1,
$$

where $\epsilon(G)$ is the number of Euler tours in $G$. Note that in the right-hand side, $\epsilon(G)=0$ unless $G$ is connected and balanced, and hence strongly connected. A digraph is called balanced if $\operatorname{deg}^{+}(v)=$ $\operatorname{deg}^{-}(v)$ for each vertex $v$.

Acknowledgements The author is grateful to Professor Kefeng Liu for his kind help over many years. The author benefited a lot from Professor Shing-Tung Yau's seminars. The author thanks Professor Xiaonan Ma for helpful comments. The author thanks the referee for very helpful suggestions which greatly improved the presentation of the paper.

\section{TENSOR CALCULUS ON KÄHLER MANIFOLDS}

Let $(M, g)$ be a Kähler manifold of dimension $n$. Locally the Kähler form is given by

$$
\omega_{g}=\frac{\sqrt{-1}}{2 \pi} \sum_{i, j=1}^{n} g_{i \bar{j}} d z_{i} \wedge d z_{\bar{j}}
$$

We will use the Einstein summation convention. The indices $i, j, k, \ldots$ run from 1 to $n$, while Greek indices $\alpha, \beta, \gamma$ may represent either $i$ or $\bar{i}$. Let $\operatorname{det} g$ be the determinant of the Hermitian matrix $\left(g_{i \bar{j}}\right)$ and $\left(g^{i \bar{j}}\right)$ be the inverse of the matrix $\left(g_{i \bar{j}}\right)$. We also use the notation

$$
g_{j \bar{k} \alpha_{1} \alpha_{2} \ldots \alpha_{m}}:=\partial_{\alpha_{1} \alpha_{2} \ldots \alpha_{m}} g_{j \bar{k}} .
$$

The curvature tensor is defined as

$$
R_{i \bar{j} k \bar{l}}=-g_{i \bar{j} k \bar{l}}+g^{m \bar{p}} g_{m \bar{j} \bar{l}} g_{i \bar{p} k} .
$$

The Ricci tensor is

$$
R_{i \bar{j}}=g^{k \bar{l}} R_{i \bar{j} k \bar{l}}=-\partial_{i} \partial_{\bar{j}} \log (\operatorname{det} g)
$$

and the scalar curvature is the trace of the Ricci curvature

$$
\rho=g^{i \bar{j}} R_{i \bar{j}} .
$$

The covariant derivative of a tensor field $T_{\beta_{1} \ldots \beta_{q}}^{\alpha_{1} \ldots \alpha_{p}}$ is defined by

$$
T_{\beta_{1} \ldots \beta_{q} ; \gamma}^{\alpha_{1} \ldots \alpha_{p}}=\partial_{\gamma} T_{\beta_{1} \ldots \beta_{q}}^{\alpha_{1} \ldots \alpha_{p}}-\sum_{i=1}^{q} \Gamma_{\gamma \beta_{i}}^{\delta} T_{\beta_{1} \ldots \beta_{i-1} \delta \beta_{i+1} \ldots \beta_{q}}^{\alpha_{1} \ldots \alpha_{p}}+\sum_{j=1}^{p} \Gamma_{\delta \gamma}^{\alpha_{j}} T_{\beta_{1} \ldots \beta_{q}}^{\alpha_{1} \ldots \alpha_{j-1} \delta \alpha_{j+1} \ldots \alpha_{p}}
$$


where the Christoffel symbols $\Gamma_{\beta \gamma}^{\alpha}=0$ except for

$$
\Gamma_{j k}^{i}=g^{i \bar{l}} g_{j \bar{l} k}, \quad \Gamma_{\bar{j} \bar{k}}^{\bar{i}}=g^{l \bar{l}} g_{l \bar{j} \bar{k}} .
$$

Lemma 2.1. For tensors in Kähler geometry, we have the following identities:

i) The Kähler metric g satisify

$$
\partial_{i} g_{j \bar{k}}=\partial_{j} g_{i \bar{k}}, \quad \partial_{\bar{l}} g_{j \bar{k}}=\partial_{\bar{k}} g_{j \bar{l}} .
$$

ii) The derivative of $g^{m \bar{l}}$ satisfy

$$
\partial_{\alpha} g^{m \bar{l}}=-g^{p \bar{l}} g^{m \bar{q}} g_{p \bar{q} \alpha} .
$$

iii) The derivative of $\operatorname{det} g$ satisfy

$$
\partial_{\alpha} \operatorname{det} g=\operatorname{det} g \cdot g^{m \bar{l}} g_{m \bar{l} \alpha} .
$$

iv) The curvature tensor satisfy the first Bianchi identity

$$
R_{i \bar{j} k \bar{l}}=R_{i \bar{l} k \bar{j}}=R_{k \bar{j} i \bar{l}} .
$$

v) The covariant derivatives of the curvature tensor satisfy the second Bianchi identity

$$
R_{i \bar{j} k \bar{l} ; m}=R_{m \bar{j} k \bar{l} ; i}=R_{i \bar{j} m \bar{l} ; k}, \quad R_{i \bar{j} k \bar{l} ; \bar{p}}=R_{i \bar{p} k \bar{l} ; \bar{j}}=R_{i \bar{j} k \bar{p} \bar{l} \bar{l}} .
$$

vi) The Ricci formula gives the difference when we interchange two covariant derivative indices

$$
T_{\beta_{1} \ldots \beta_{q} ; \bar{j}}^{\alpha_{1} \ldots \alpha_{p}}-T_{\beta_{1} \ldots \beta_{q} ; \bar{j} i}^{\alpha_{1} \ldots \alpha_{p}}=\sum_{k=1}^{q} R_{\beta_{k} i \bar{j}}^{\gamma} T_{\beta_{1} \ldots \beta_{k-1} \gamma \beta_{k+1} \ldots \beta_{q}}^{\alpha_{1} \ldots \alpha_{p}}-\sum_{l=1}^{p} R_{\gamma i \bar{j}}^{\alpha_{l}} T_{\beta_{1} \ldots \beta_{q}}^{\alpha_{1} \ldots \alpha_{l-1} \gamma \alpha_{l+1} \ldots \alpha_{p}},
$$

where $R_{\overline{l i} \bar{j}}^{\bar{k}}=-g^{m \bar{k}} R_{m \bar{i} \bar{j}}, R_{l i \bar{j}}^{k}=g^{k \bar{m}} R_{l \bar{m} i \bar{j}}$ and $R_{\overline{l i} \bar{j}}^{k}=R_{l i \bar{j}}^{\bar{k}}=0$.

Recall that around each point $x$ on a Kähler manifold, there exists a normal coordinate such that

$$
g_{i \bar{j}}(x)=\delta_{i j}, \quad g_{i \bar{j} k_{1} \ldots k_{r}}(x)=g_{i \bar{j} \bar{l}_{1} \ldots \bar{l}_{r}}(x)=0
$$

for all $r \leq N \in \mathbb{N}$, where $N$ can be chosen arbitrary large.

Remark 2.2. Fix a normal coordinate around $x$ on a Kähler manifold. By (11) and (14), it is not difficult to see inductively that any covariant derivative $R_{i \bar{j} k \bar{l} ; \alpha_{1} \ldots \alpha_{r}}$ is canonically equal to a polynomial of $g_{a \bar{b} \alpha}$ and their partial derivatives. By (22), any monomial containing $g_{i \bar{j} k_{1} \ldots k_{r}}$ or $g_{i \bar{j} \bar{l}_{1} \ldots \bar{l}_{r}}$ vanishes at $x$. For example

$$
\begin{aligned}
& R_{i \bar{j} k \bar{l}}(x)=-g_{i \bar{j} k \bar{l}}(x), \\
& R_{i \bar{j} k \bar{l} ; p \bar{q}}(x)=-g_{i \bar{j} k \bar{l} p \bar{q}}(x)+g^{s \bar{t}}\left(g_{p \bar{j} s \bar{l}} g_{i \bar{q} k \bar{t}}+g_{k \bar{j} s \bar{l}} g_{p \bar{q} i \bar{t}}+g_{i \bar{j} s \bar{l}} g_{k \bar{q} p \bar{t}}\right)(x), \\
& R_{i \bar{j} k \bar{l} ; p_{1} \ldots p_{r}}(x)=-g_{i \bar{j} k \bar{l} p_{1} \ldots p_{r}}(x), \quad \forall r \geq 1, \\
& R_{i \bar{j} k \bar{l} ; \bar{p}_{1} \ldots \bar{p}_{r}}(x)=-g_{i \bar{j} k l \bar{p}_{1} \ldots \bar{p}_{r}}(x), \quad \forall r \geq 1 .
\end{aligned}
$$

Note that these identities hold only at the point $x$. However they can easily recover the original identities (hold in a neighborhood of $x$ ), since from (11) and (14), we see that $g_{i \bar{j} k \bar{l} \beta_{1} \ldots \beta_{r}}$ can be inductively expressed as a canonical polynomial of covariant derivatives of curvature tensors, denoted by $D\left(g_{i \bar{j} k \bar{l} \beta_{1} \ldots \beta_{r}}\right)$. For example

$$
\begin{gathered}
D\left(g_{i \bar{j} k \bar{l}}\right)=-R_{i \bar{j} k \bar{l}}, \\
D\left(g_{i \bar{j} k \bar{l} p \bar{q}}\right)=-R_{i \bar{j} k \bar{l} ; p \bar{q}}+g^{s \bar{t}}\left(R_{p \bar{j} s \bar{l}} R_{i \bar{q} k \bar{t}}+R_{k \bar{j} s \bar{l}} R_{p \bar{q} i \bar{t}}+R_{i \bar{j} s \bar{l}} R_{k \bar{q} p \bar{t}}\right) .
\end{gathered}
$$

In general, we can inductively get

$$
D\left(g_{i \bar{j} k \bar{l} \beta_{1} \ldots \beta_{r}}\right)=-R_{i \bar{j} k \bar{l} ; \beta_{1} \ldots \beta_{r}}+\text { covariant derivatives of lower order. }
$$

In Section [5] this observation will allow us to store Weyl invariants as polynomials of $g_{a \bar{b}} c \bar{d}$ and their derivatives. The advantage is that we do not need to deal with the problem of exchanging indices as in the Ricci formula (21). 

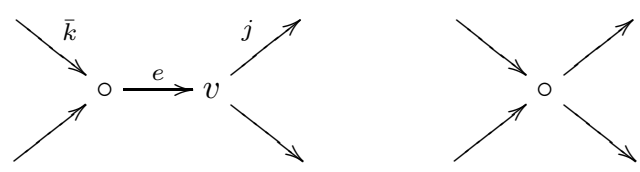

Figure 1. The atomic admissible tree and a trivial admissible tree

We will now define the concept of an admissible tree, which is a directed tree with half-edges. We call the two trees in Figure 1 primitive admissible trees.

We can define three types of actions by an outward half-edge $i$ or an inward half-edge $\bar{i}$ on a directed tree with half-edges. Let us take the left-hand primitive admissible tree as an example:

(1) The action on a vertex $v$ is to add the half edge to the vertex (see Figure 2).

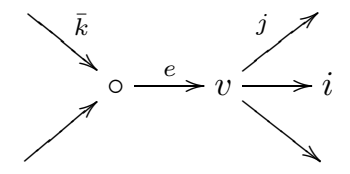

or

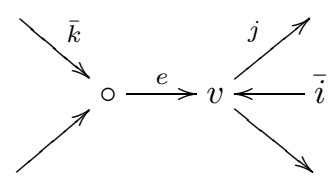

FiguRe 2. Action on a vertex $v$

(2) The action on a half-edge with the same direction (i.e. $i$ can only act on outward half-edges and $\bar{i}$ only act on inward half-edges) is to put them together on a new vertex (see Figure 3).

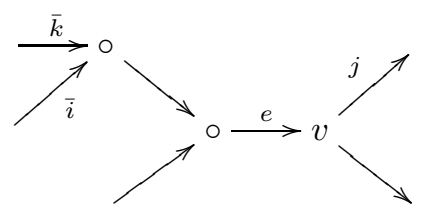

or

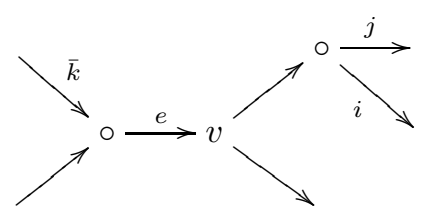

Figure 3. Action on a half-edge

(3) The action on an edge $e$ is to generate a new vertex to split $e$ and add the half-edge to the new vertex (see Figure 4).

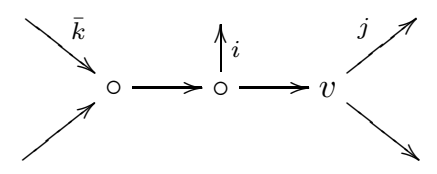

or

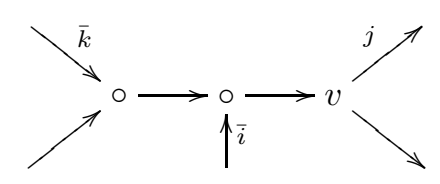

Figure 4. Action on an edge $e$

For any two trees $T_{1}$ and $T_{2}$, we can join them by gluing an outward half-edge of $T_{1}$ to an inward half-edge of $T_{2}$, or vice versa.

Definition 2.3. An admissible tree (with half-edges) is defined to be the join of finite number of trees which can be obtained by a finite number of the above three actions on the primitive admissible trees in Figure 1. For an admissible tree, we usually label its outward half-edges and inward half-edges with distinct indices $\{i, j, k, \ldots\}$ and $\{\bar{l}, \bar{m}, \bar{p}, \ldots\}$ respectively.

An admissible tree is called decomposable if we can cut an edge to get two admissible trees (see Figure 5). Otherwise, it is called indecomposable. 


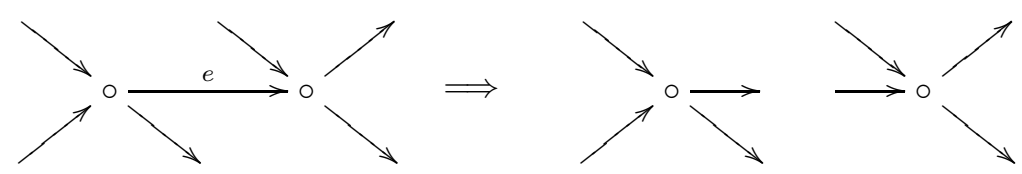

Figure 5. Decomposition by cutting an edge $e$

Definition 2.4. For any $g_{i \bar{j} k \bar{l} \beta_{1} \ldots \beta_{r}}$, as in remark 2.2, there exists a canonical polynomial of covariant derivatives of curvature tensor, denoted by $D\left(g_{i \bar{j} k \bar{l} \beta_{1} \ldots \beta_{r}}\right)$, that coincides with $g_{i \bar{j} k \bar{l} \beta_{1} \ldots \beta_{r}}$ at the center of the normal coordinate. By (11) and (14), $D\left(g_{i \bar{j} k \bar{l} \beta_{1} \ldots \beta_{r}}\right)$ may be written as a unique universal polynomial of $g_{a \bar{b} \gamma_{1} \ldots \gamma_{t}}$ with $t \geq 1$.

For example,

$$
D\left(g_{i \bar{j} k \bar{l}}\right)=g_{i \bar{j} k \bar{l}}-g^{m \bar{p}} g_{m \bar{j} \bar{l}} g_{i k \bar{p}}
$$

Obviously, the above identity can be expressed in terms of admissible trees

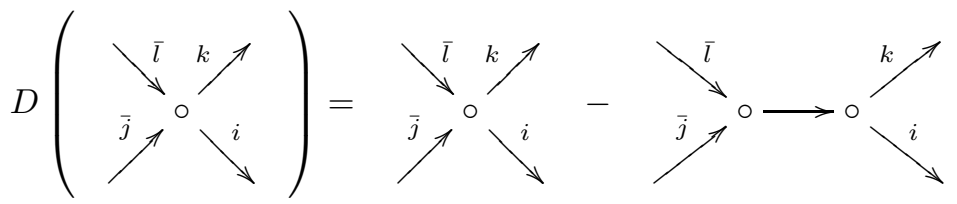

The map $D$ can be naturally extended to be defined on any admissible tree with both indegree and outdegree $\geq 2$ at each vertex. We simply apply $D$ to each vertex while keeping index pairings and expanding linearly.

Let $\mathcal{T}_{m}\left(\alpha_{1}, \ldots, \alpha_{r}\right)$ denote the set of all indecomposable admissible trees with $m$ vertices and $r$ half edges labeled by the set of indices $\left\{\alpha_{1}, \ldots, \alpha_{r}\right\}$.

Lemma 2.5. We have

$$
D\left(g_{i \bar{j} k \bar{l} \beta_{1} \ldots \beta_{r}}\right)=\sum_{m=1}^{r+2} \sum_{T \in \mathcal{T}_{m}\left(i, \bar{j}, k, \bar{l}, \beta_{1}, \ldots, \beta_{r}\right)}(-1)^{m+1} T .
$$

Proof. (Sketch) We first note that equation (17) corresponds to the action on edges in Figure 4 and the multiplication by $\Gamma$ in equation (14) corresponds to the action on half-edges in Figure 3 .

By (11), we have

$$
R_{i \bar{j} k \bar{l} \beta_{1} \ldots \beta_{r}}=-g_{i \bar{j} k \bar{l} \beta_{1} \ldots \beta_{r}}+\left(g^{m \bar{p}} g_{m \bar{j} \bar{l}} g_{i k \bar{p}}\right)_{\beta_{1} \ldots \beta_{r}}
$$

The left-hand side is a derivative of $R_{i \bar{j} k \bar{l}}$. By induction, we can see that $D\left(\left(g^{m \bar{p}} g_{m \bar{j} \bar{l}} g_{i k \bar{p}}\right)_{\beta_{1} \ldots \beta_{r}}\right)$ is a sum over all decomposable admissible trees with the set of indices $\left\{i, \bar{j}, k, \bar{l}, \beta_{1}, \ldots, \beta_{r}\right\}$ such that no irreducible component of the tree can contain indices from both of the two sets $\{i, k\}$ and $\{\bar{j}, \bar{l}\}$. We can apply (14) inductively to see that all trees from $R_{i \bar{j} k \bar{l}} \beta_{1} \ldots \beta_{r}$ are admissible and get the desired equation (25).

\section{The local Bergman kernel and the Berezin transform}

In this section, we consider only the case that $\mathcal{E}=\mathbb{C}$. So the Bergman kernel $B_{m}(x)$ is a scalar function on $M$.

Now we introduce the local Bergman kernel following [15]. Let $\Omega$ be a bounded domain in $\mathbb{C}^{n}$ and $\Phi$ be a Kähler potential for a Kähler metric $g$ on $\Omega$ satisfying

$$
\omega_{g}=\frac{\sqrt{-1}}{2 \pi} \partial \bar{\partial} \Phi
$$

To simplify, we may assume $\Omega$ is equipped with a normal coordinate. 
Let $\Phi(x, y)$ be an almost analytic extension to a neighborhood of the diagonal, i.e. $\bar{\partial}_{x} \Phi$ and $\partial_{y} \Phi$ vanish to infinite order for $x=y$ (cf. [6]). We can assume $\overline{\Phi(x, y)}=\Phi(y, x)$. Consider the real valued function

$$
D(x, y)=\Phi(x, x)+\Phi(y, y)-\Phi(x, y)-\Phi(y, x)
$$

which is called the Calabi diastatic function [8. It is easily seen that the function $D(x, y) \geq 0$ and $D(x, y)=0$ if and only $x=y$.

We need the following important result of Engliš [15].

Theorem 3.1. (Engliš) There is an asymptotic expansion for the Laplace integral

$$
\int_{\Omega} f(y) e^{-m D(x, y)} \frac{\omega_{g}^{n}(y)}{n !} \sim \frac{1}{m^{n}} \sum_{j \geq 0} m^{-j} R_{j}(f)(x),
$$

where $R_{j}: C^{\infty}(\Omega) \rightarrow C^{\infty}(\Omega)$ are explicit differential operators defined by

$$
R_{j} f(x)=\left.\frac{1}{\operatorname{det} g} \sum_{k=j}^{3 j} \frac{1}{k !(k-j) !} L^{k}\left(f \operatorname{det} g S^{k-j}\right)\right|_{y=x},
$$

where $L$ is the (constant-coefficient) differential operator

$$
L f(y)=g^{i \bar{j}}(x) \partial_{i} \partial_{\bar{j}} f(y)
$$

and the function $S(x, y)$ satisfies

$$
\begin{gathered}
S=\partial_{\alpha} S=\partial_{\alpha \beta} S=\partial_{i_{1} i_{2} \ldots i_{m}} S=\partial_{\bar{i}_{1} \bar{i}_{2} \ldots \bar{i}_{m}} S=0 \quad \text { at } y=x, \\
\left.\partial_{i \bar{j} \alpha_{1} \alpha_{2} \ldots \alpha_{m}} S\right|_{y=x}=-\partial_{\alpha_{1} \alpha_{2} \ldots \alpha_{m}} g_{i \bar{j}}(x) .
\end{gathered}
$$

In particular, we have

$$
\left\{\begin{aligned}
R_{0}=i d & \\
R_{1}(f)= & \Delta f-\frac{1}{2} f \rho . \\
R_{2}(f)= & \frac{1}{2} \Delta^{2} f-\frac{1}{2} L_{R i c} f-\frac{\rho}{2} \Delta f-\frac{1}{2}\left(\rho_{i} f_{; i}+\rho_{\bar{i}} f_{; \bar{i}}\right) \\
\quad & \quad\left(\frac{1}{3} \Delta \rho-\frac{1}{8} \rho^{2}-\frac{1}{6}|R i c|^{2}+\frac{1}{24}|R|^{2}\right) f .
\end{aligned}\right.
$$

Here $L_{R i c} f=R_{j \bar{i}} f_{; i \bar{j}},|R i c|^{2}=R_{i \bar{j}} R_{j \bar{i}},|R|^{2}=R_{i \bar{j} k \bar{l}} R_{j \bar{i} l \bar{k}}$. Note that our convention of curvatures $R_{i \bar{j} k \bar{l}}, R_{i \bar{j}}, \rho$ all differ by a minus sign with that of [15].

We need the following extension of Tian-Yau-Zelditch asymptotic expansion [9, 16, 19].

Theorem 3.2. Let $M$ be a compact complex manifold endowed with a polarized Kähler metric $g$. Let $B_{m}(x, y)$ denote an almost analytic extension of $B_{m}(x)$ to an open neighborhood, say $U \times U$ of the diagonal. Then, for $U$ sufficiently small, $B_{m}(x, y)$ admits an asymptotic expansion (as $m \rightarrow+\infty$ ) of the form:

$$
B_{m}(x, y) \sim \sum_{j \geq 0} a_{j}(x, y) m^{n-j} .
$$

For $\alpha>0$, consider the weighted Bergman space of all holomorphic function on $\Omega$ square-integrable with respect to the measure $e^{-\alpha \Phi} \frac{w_{g}^{n}}{n !}$.

We denote by $K_{\alpha}(x, y)$ the reproducing kernel. As pointed out by Engliš [15, it is often the case that the following holds. (e.g. if $\left(\Omega, g_{i \bar{j}}\right)$ is a bounded symmetric with the invariant metric or whenever $\Omega$ is strongly pseudoconvex with real analytic boundary.)

(1) $K_{\alpha}(x, y)$ has an asymptotic expansion in a small neighborhood of the diagonal

$$
K_{\alpha}(x, y) \sim e^{\alpha \Phi(x, y)} \sum_{k=0}^{\infty} b_{k}(x, y) \alpha^{n-k} .
$$

(2) For any neighborhood $U$ of a point $x \in \Omega$ and a bounded measurable function $f$,

$$
\int_{\Omega \backslash U} f(y) \frac{\left|K_{\alpha}(x, y)\right|^{2}}{K_{\alpha}(x, x)} e^{-\alpha \Phi(y)} \frac{w_{g}^{n}(y)}{n !}=o\left(\alpha^{-k}\right), \quad \forall k \geq 1 .
$$


Denote $b_{k}(x, x)$ by $b_{k}(x)$.

Theorem 3.3. For any $k \geq 0$, we have $a_{k}(x)=b_{k}(x)$.

This theorem means that the global Bergman kernel can be approximated by the local Bergman kernel. An analytic proof of this theorem can be found in [2]. Here we present a simple proof which is implicit in 22 .

The Berezin transform is the integral operator

$$
I_{\alpha} f(x)=\int_{\Omega} f(y) \frac{\left|K_{\alpha}(x, y)\right|^{2}}{K_{\alpha}(x, x)} e^{-\alpha \Phi(y)} \frac{w_{g}^{n}(y)}{n !} .
$$

At any point for which $K_{\alpha}(x, x)$ invertible, the integral converges for each bounded measurable function $f$ on $\Omega$.

Assume that (28) and (29) hold, then the Berezin transform has an asymptotic expansion (see [15])

$$
I_{\alpha} f(x)=\sum_{k=0}^{\infty} Q_{k} f(x) \alpha^{-k}
$$

where $Q_{k}$ are differential operators.

We have that for $(x, y)$ near the diagonal,

$$
\frac{\left|K_{\alpha}(x, y)\right|^{2}}{K_{\alpha}(x, x)} e^{-\alpha \Phi(y)}=e^{-\alpha D(x, y)} \sum_{k=0}^{\infty} \tilde{b}_{k}(x, y) \alpha^{n-k},
$$

where $\tilde{b}_{0}=1, \tilde{b}_{1}(x, y)=b_{1}(x, y)+b_{1}(y, x)-b_{1}(x, x)$, etc.

Fix a bounded neighborhood $U$ of $x$ such that (32) holds. Applying Theorem 3.1 , we get

$$
Q_{k} f(x)=\left.\sum_{j=0}^{k} R_{j}\left(\tilde{b}_{k-j}(x, y) f(y)\right)\right|_{y=x},
$$

where the operators $R_{j}$ apply to the $y$-variable.

Since $Q_{k} f=0$ when $k>0$ and $f$ is analytic or anti-analytic, setting $f=1$ in (33), we get

$$
b_{k}(x)=-\left.\sum_{j=1}^{k} R_{j}\left(\tilde{b}_{k-j}(x, y)\right)\right|_{y=x}, \quad k \geq 1 .
$$

The following result from quantization of Kähler manifolds can be found in [7, 22].

Theorem 3.4. In the same hypothesis of Theorem 3.2, we have a globally defined function on $M$.

$$
\psi_{m}(x, y)=\frac{e^{-m D(x, y)}\left|B_{m}(x, y)\right|^{2}}{B_{m}(x) B_{m}(y)}
$$

Then

$$
\int_{M} \psi_{m}(x, y) B_{m}(y) \frac{w_{g}^{n}(y)}{n !}=1
$$

Moreover, for any neighborhood $U$ of $x \in M$ and every smooth function $f$ on $M$.

$$
\int_{M \backslash U} \psi_{m}(x, y) B_{m}(y) \frac{w_{g}^{n}(y)}{n !}=o\left(m^{-k}\right), \quad \forall k \geq 1 .
$$

From (35) and Theorem 3.1, we get

$$
\left.\sum_{j=0}^{k} R_{j}\left(\tilde{a}_{k-j}(x, y)\right)\right|_{y=x}=0,
$$

where $\tilde{a}_{0}=1, \tilde{a}_{1}(x, y)=a_{1}(x, y)+a_{1}(y, x)-a_{1}(x)$, etc. Note that this is exactly the same recursive equation as (34). Since $a_{0}(x)=b_{0}(x)=1$, we must have $a_{k}(x)=b_{k}(x)$ for all $k \geq 0$. So we conclude the proof of Theorem 3.3 . 


\section{The Bergman KeRnel of VeCtor BUNDLES}

Let $h(x)$ be a Hermitian metric on $\Omega \times \mathbb{C}^{r}$. For $\alpha>0$, consider the weighted Bergman space of all holomorphic function $f: \Omega \rightarrow \mathbb{C}^{r}$ square-integrable with respect to the norm

$$
\|f\|^{2}=\int_{\Omega}\langle f, f\rangle_{h} e^{-\alpha \Phi} \frac{w_{g}^{n}}{n !} .
$$

Now the Bergman kernel $K_{\alpha}(x, y)$ takes value in matrices. The results in the last section could be extended without difficulty to the matrix case.

By the property of reproducing kernels, we have for $v \in \mathbb{C}^{r}$,

$$
\left\langle K_{\alpha}(x, x) f(x), v\right\rangle=\int_{\Omega}\left\langle K_{\alpha}(x, y) f(y), K_{\alpha}(x, y) v\right\rangle e^{-\alpha \phi(y)} g(y) d y .
$$

We take $f(x)$ to be a constant function and take the inner product to be the Hermitian metric $h_{\alpha \bar{\beta}}$. Let $H=h_{\alpha}^{\beta} \in \Gamma(\operatorname{End}(\mathcal{E}))$ and apply Theorem 3.1 to (37), we get

$$
a_{k}(x)=\left.\sum_{\ell+i+j=k} R_{\ell}\left(a_{i}(x, y) a_{j}(y, x) H\right)\right|_{y=x}
$$

Remark 4.1. The connection of Engliš' work and the asymptotic expansion of Tian-Yau-Zelditch was studied by Loi [22, who obtained the formula (38) when $\mathcal{E}=\mathbb{C}$ using Theorem 3.4.

From the curvature equation

$$
\Theta_{i \bar{j}} H=-H_{i \bar{j}}+\partial_{i} H \partial_{\bar{j}} H
$$

we have

$$
\begin{aligned}
\left(H_{i \bar{i}}\right)_{/ j \bar{j}} & =-\Theta_{i \bar{i} / j \bar{j}} H-\Theta_{i \bar{i}} H_{j \bar{j}}+H_{i \bar{j}} H_{j \bar{i}} \\
& =-\wedge \partial \bar{\partial} \wedge \Theta+\wedge \Theta \wedge \Theta+\Theta_{i \bar{j}} \Theta_{j \bar{i}} .
\end{aligned}
$$

Since $R_{0}$ is the identity operator, we have

$$
a_{k}(x)=-R_{k}(H)-\sum_{\substack{i+j=k \\ i, j \geq 1}} a_{i}(x) a_{j}(x) H-\left.\sum_{\substack{\ell+i+j=k \\ 1 \leq \ell \leq k-1}} R_{\ell}\left(a_{i}(x, y) a_{j}(y, x) H\right)\right|_{y=x} .
$$

Then it is not difficult to compute the first few terms

$$
a_{1}(x)=-\Delta H+\frac{\rho}{2} H=\frac{\rho}{2} I+\wedge \Theta .
$$

Note that in the second equation, we used $H(0)=I$.

For $k=2$, we need a little more work.

$$
a_{2}(x)=-R_{2}(H)-a_{1}(x)^{2} H-\left.R_{1}\left(a_{1}(x, y) H\right)\right|_{y=x}-\left.R_{1}\left(a_{1}(y, x) H\right)\right|_{y=x} .
$$

We compute terms in the right-hand side one by one. By formula (39), we have

$$
\begin{aligned}
R_{2}(H)= & \frac{1}{2} \Delta \Delta H-\frac{1}{2} R_{i \bar{j}} H_{j \bar{i}}-\frac{\rho}{2} \Delta H-\left(\frac{1}{3} \Delta \rho-\frac{1}{8} \rho^{2}-\frac{1}{6}|R i c|^{2}+\frac{1}{24}|R|^{2}\right) H \\
= & \left(-\frac{1}{2} \wedge \partial \bar{\partial} \wedge \Theta+\frac{1}{2} \Theta_{i \bar{j}} \Theta_{j \bar{i}}+\frac{1}{2} \wedge \Theta \wedge \Theta\right)+\frac{1}{2} R_{i \bar{j}} \Theta_{j \bar{i}}+\frac{\rho}{2} \wedge \Theta \\
& -\left(\frac{1}{3} \Delta \rho-\frac{1}{8} \rho^{2}-\frac{1}{6}|R i c|^{2}+\frac{1}{24}|R|^{2}\right) I .
\end{aligned}
$$

Substituting $a_{1}(x)$ computed in (41), we have

$$
a_{1}(x)^{2} H=\frac{\rho^{2}}{4} I+\rho \wedge \Theta+\wedge \Theta \wedge \Theta .
$$

From almost-analyticity of $a_{1}(x, y)$, we get

$$
\begin{aligned}
\left.R_{1}\left(a_{1}(x, y) H\right)\right|_{y=x} & =\left.\Delta\left(a_{1}(x, y) H\right)\right|_{y=x}-\frac{\rho}{2} a_{1}(x) H \\
& =a_{1}(x) \Delta H-\frac{\rho}{2} a_{1}(x)
\end{aligned}
$$




$$
\begin{aligned}
& =-\frac{\rho}{2} \wedge \Theta-\wedge \Theta \wedge \Theta-\frac{\rho^{2}}{4} I-\frac{\rho}{2} \wedge \Theta \\
& =-\frac{\rho^{2}}{4} I-\wedge \Theta \wedge \Theta-\rho \wedge \Theta .
\end{aligned}
$$

Similarly, $\left.R_{1}\left(a_{1}(y, x) H\right)\right|_{y=x}=-\frac{\rho^{2}}{4} I-\wedge \Theta \wedge \Theta-\rho \wedge \Theta$.

Summing up the above computation, we arrive at the desired value of $a_{2}(x)$,

$$
\begin{aligned}
a_{2}(x)= & \frac{1}{2} \Delta \wedge \Theta+\frac{1}{2}(\wedge \Theta)^{2}+\frac{\rho}{2} \wedge \Theta-\frac{1}{2} \Theta_{i \bar{j}} \Theta_{j \bar{i}}-\frac{1}{2} R_{i \bar{j}} \Theta_{j \bar{i}} \\
& +\left(\frac{1}{3} \Delta \rho+\frac{1}{8} \rho^{2}-\frac{1}{6}|R i c|^{2}+\frac{1}{24}|R|^{2}\right) I,
\end{aligned}
$$

where $\Delta \wedge \Theta=\wedge \partial \bar{\partial} \wedge \Theta$.

Definition 4.2. (Lu [23]) Let $P$ be a $d$-th order covariant derivative of $R_{i \bar{j} k \bar{l}}, R_{i \bar{j}}, \rho, \Theta, \wedge \Theta$. Define the weight and the order of $P$ to be the numbers $w(P)=\left(1+\frac{d}{2}\right)$ and $\operatorname{ord}(P)=\frac{d}{2}$ respectively. The weight and order functions can be extended additively to monomials of curvatures and their derivatives.

For example,

$$
\begin{gathered}
w\left(R_{i \bar{j} k \bar{l}}\right)=1, \quad w\left(R_{i \bar{j}, k}\right)=\frac{3}{2}, \quad w\left(R_{i \bar{j}, k} R_{i \bar{j} k \bar{l}}\right)=\frac{5}{2}, \\
\operatorname{ord}\left(R_{i \bar{j} k \bar{l}}\right)=0, \quad \operatorname{ord}\left(\rho_{i} R_{i \bar{j} k \bar{l}}\right)=\frac{1}{2}, \quad \operatorname{ord}(\Delta \wedge \Theta)=1 .
\end{gathered}
$$

In the following, the word "leading term" will mean the sum of terms with the highest order.

Lemma 4.3. The leading term in $a_{k}$ is $\frac{k}{(k+1) !} \Delta^{k-1} \rho I+\frac{1}{k !} \Delta^{k-1} \wedge \Theta$.

Proof. Let $u_{k}$ denote the coefficient of $\Delta^{k-1} \rho$ in $R_{k}(1)$. Then from (27),

$$
R_{k}(1)=\left.\frac{1}{\operatorname{det} g} \sum_{i=k}^{2 k} \frac{1}{i !(i-k) !} L^{i}\left(\operatorname{det} g S^{i-k}\right)\right|_{y=0},
$$

here we take normal coordinate system around point $x=0$ on the Kähler manifold $M$. It is not difficult to see that the only terms that may contribute to $\Delta^{k-1} \rho$ must have $i=k$ or $i=k+1$. So we have

$$
u_{k}=-\frac{1}{k !}+\frac{1}{(k+1) !}=-\frac{k}{(k+1) !} .
$$

From (40), we see that the coefficient of $\Delta^{k-1} \rho$ in $a_{k}$ equals $-u_{k}=\frac{k}{(k+1) !}$.

Similarly, we can prove that the coefficient of $\Delta^{k-1} \wedge \Theta$ in $a_{k}$ equals $\frac{1}{k !}$.

Remark 4.4. Lemma 4.3 was obtained by Liu and Lu 21] using peak section method, improving the $\mathcal{E}=\mathbb{C}$ case proved in $[24]$.

Let $M=M_{1} \times M_{2}$ a product of two projective manifolds equipped with the product Kähler metric and the twisted bundles $\mathcal{E}^{(1)}(m) \otimes \mathcal{E}^{(2)}(m)$. Denote the Bergman kernel of $M$ by $B_{m}^{(M)}$. The following fact is well-known.

Lemma 4.5. We have $B_{m}^{(M)}\left(x_{1}, x_{2}\right)=B_{m}^{\left(M_{1}\right)}\left(x_{1}\right) B_{m}^{\left(M_{2}\right)}\left(x_{2}\right)$.

Proof. We just need to note that if $\left(s_{1}, \cdots, s_{i}, \cdots\right)$ and $\left(t_{1}, \cdots, t_{j}, \cdots\right)$ are orthonormal basis of $\mathcal{E}^{(1)}(m)$ and $\mathcal{E}^{(2)}(m)$ respectively, then $\left(s_{1} t_{1}, \cdots, s_{i} t_{j}, \cdots\right)$ is an orthonormal basis of $\mathcal{E}^{(1)}(m) \otimes \mathcal{E}^{(2)}(m)$.

Consider the semigroup $N^{\infty}$ of sequences $\mathbf{d}=\left(d_{1}, d_{2}, \ldots\right)$ where $d_{i}$ are nonnegative integers and $d_{i}=0$ for sufficiently large $i$. We sometimes also use $\left(1^{d_{1}} 2^{d_{2}} \ldots\right)$ to denote $\mathbf{d}$. We will use the following notation:

$$
|\mathbf{d}|:=\sum_{i \geq 1} i \cdot d_{i}, \quad\|\mathbf{d}\|:=\sum_{i \geq 1} d_{i}, \quad \mathbf{d} !=\prod_{i \geq 1} d_{i} !, \quad \mathbf{c} \cdot \mathbf{d}:=\sum_{i \geq 1} c_{i} d_{i} .
$$


Proposition 4.6. Let $\mathbf{c}, \mathbf{d}, \mathbf{e} \in N^{\infty}$ and $|\mathbf{c}|+\mathbf{d} \cdot \mathbf{e}+\|\mathbf{e}\|=k$. Let $u(\mathbf{c}, \mathbf{d}, \mathbf{e})$ denote the coefficient of the weight $k$ monomial ( where $d_{j} \neq d_{j+1}, \forall j \geq 1$ )

$$
\prod_{i \geq 1}\left(\Delta^{i-1} \rho\right)^{c_{i}} \prod_{j \geq 1}\left(\Delta^{d_{j}} \wedge \Theta\right)^{e_{j}}
$$

in $a_{k}$. Then we have

$$
u(\mathbf{c}, \mathbf{d}, \mathbf{e})=\frac{1}{\mathbf{c} !} \prod_{i \geq 1}\left(\frac{i}{(i+1) !}\right)^{c_{i}} \cdot \frac{1}{\mathbf{e} !} \prod_{j \geq 1}\left(\frac{1}{\left(d_{j}+1\right) !}\right)^{e_{j}} .
$$

Proof. By Lemma 4.3 and Lemma 4.5. we have the following relations:

$$
\begin{aligned}
c_{i} \cdot u(\mathbf{c}, \mathbf{d}, \mathbf{e}) & =\frac{i}{(i+1) !} u\left(\mathbf{c}-\boldsymbol{\delta}_{i}, \mathbf{d}, \mathbf{e}\right), \\
e_{j} \cdot u(\mathbf{c}, \mathbf{d}, \mathbf{e}) & =\frac{1}{\left(d_{j}+1\right) !} u\left(\mathbf{c}, \mathbf{d}, \mathbf{e}-\boldsymbol{\delta}_{j}\right),
\end{aligned}
$$

where $\boldsymbol{\delta}_{i} \in N^{\infty}$ denotes the sequence with 1 at the $i$-th place and zeros elsewhere.

From $u(\mathbf{0}, \mathbf{0}, \mathbf{0})=1$, we can recursively prove the desired formula (44) of $u(\mathbf{c}, \mathbf{d}, \mathbf{e})$.

\section{WEYL INVARIANTS AND GRAPHS}

In the rest of the paper, we will restrict to the case $\mathcal{E}=\mathbb{C}$, namely $\Theta=0$.

The $a_{k}$ 's in (4) are the so-called Weyl invariants introduced by Fefferman [17. Consider the tensor products of covariant derivatives of the curvature tensor $R_{i \bar{j} k \bar{l} ; p \cdots \bar{q}}$, e.g.

$$
R_{i j k \bar{l} ; p \bar{q}} \otimes \cdots \otimes R_{a \bar{b} c \bar{d} ; \bar{e}} .
$$

The Weyl invariants are constructed by first pairing up the unbarred indices to barred indices and then contracting all paired indices.

For the sake of brevity, Weyl invariants such as

$$
g^{i_{1} \bar{j}_{1}} g^{i_{2} \bar{j}_{2}} g^{i_{3} \bar{j}_{3}} g^{i_{4} \bar{j}_{4}} g^{i_{5} \bar{j}_{5}} R_{i_{1} \bar{j}_{1} i_{2} \bar{j}_{3} ; i_{5} \bar{j}_{4}} R_{i_{3} \bar{j}_{2} i_{4} \bar{j}_{5}}
$$

will be abbreviated as

$$
W:=R_{i_{1} \bar{i}_{1} i_{2} \bar{i}_{3} ; i_{5} \bar{i}_{4}} R_{i_{3} \bar{i}_{2} i_{4} \bar{i}_{5}},
$$

knowing that $\left(i_{k}, \bar{i}_{k}\right), 1 \leq k \leq 5$ are paired indices to be contracted.

It is useful to represent Weyl invariants as digraphs (also called quivers), namely directed graphs possibly with loops and multi-edges. We put curvature tensors as nodes and draw a directed edge from $i_{k}$ to $\bar{i}_{k}$ for each $k$. For example, the associated graph of the above Weyl invariant (45) is depicted in Figure 6.

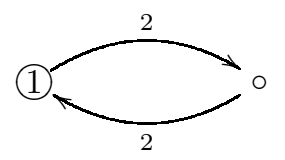

FiguRE 6 . The associated graph of $W$

In this paper, we represent a multidigraph as a weighted digraph. The weight of a directed edge is the number of multi-edges. The number attached to a vertex denotes the number of its self-loops. A vertex without loops will be denoted by a small circle. The indegree and outdegree of a vertex $v$ are

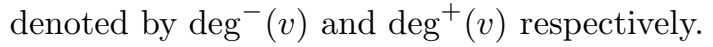

By Ricci formula, we cannot recover a Weyl invariant from its associated graph, namely different Weyl invariants may have the same associated graph. As mentioned in Remark 2.2, a remedy for the discrepancy is to express the Weyl invariant in terms of derivatives of the Kähler metric. No information will be lost and the uniqueness of the associated graph is guaranteed by (16). 
Definition 5.1. We call a vertex $v$ of a digraph $G$ semistable if we have

$$
\operatorname{deg}^{-}(v) \geq 1, \operatorname{deg}^{+}(v) \geq 1, \operatorname{deg}^{-}(v)+\operatorname{deg}^{+}(v) \geq 3 .
$$

$G$ is called semistable if each vertex of $G$ is semistable. We call $v$ stable if $\operatorname{deg}^{-}(v) \geq 2, \operatorname{deg}^{+}(v) \geq 2$. A digraph $G$ is stable if each vertex of $G$ is stable.

Let $\mathcal{G}_{r, s}$ denote the set of stable digraphs with $r$ vertices and $s$ edges. The set of semistable and stable graphs will be denoted by $\mathcal{G}^{s s}$ and $\mathcal{G}$ respectively. The associated graph of a Weyl invariant lies in $\mathcal{G}$. For any $G \in \mathcal{G}_{r, s}$, its weight $w(G)$ is defined to be $s-r$. We denote by $\mathcal{G}^{s s}(k)$ and $\mathcal{G}(k)$ respectively the set of semistable and stable digraphs with weight $k$. Let $\mathcal{G}_{\text {con }}(k)$ and $\mathcal{G}_{\text {scon }}(k)$ respectively be the set of connected and strongly connected graphs in $\mathcal{G}(k)$. We also define a special set of graphs:

$$
\Lambda(k)=\left\{G \in \mathcal{G}_{\text {scon }}(k) \mid 1 \text { is not an eigenvalue of } A(G)\right\} .
$$

This set is of interest in view of Corollary 6.8 We have computed the cardinalities of these sets when $k \leq 5$ in Table 1 .

TABLE 1. Numbers of stable graphs

\begin{tabular}{|c||c|c|c|c|c|}
\hline$k$ & 1 & 2 & 3 & 4 & 5 \\
\hline$|\mathcal{G}(k)|$ & 1 & 4 & 15 & 82 & 589 \\
\hline$\left|\mathcal{G}_{\text {con }}(k)\right|$ & 1 & 3 & 11 & 61 & 474 \\
\hline$\left|\mathcal{G}_{\text {scon }}(k)\right|$ & 1 & 3 & 10 & 51 & 373 \\
\hline$|\Lambda(k)|$ & 1 & 3 & 9 & 45 & 316 \\
\hline
\end{tabular}

It is not difficult to see that $\mathcal{G}(k)=\cup_{j=1}^{k} \mathcal{G}_{j, j+k}$ for any $k \geq 1$.

Remark 5.2. It shall be interesting to find formulae, closed or recursive, for the number of graphs in the above sets.

We can write the coefficient $a_{k}$ as a polynomial of $g_{i \bar{j} \beta_{1} \ldots \beta_{r}}, r \geq 1$, and consequently as a sum over graphs in $\mathcal{G}^{s s}(k)$,

$$
a_{k}(x)=\sum_{G \in \mathcal{G}^{s s}(k)} z(G) \cdot G, \quad z(G) \in \mathbb{Q} .
$$

So we may regard $z$ as a map from $\mathcal{G}^{s s}$ to $\mathbb{Q}$. By Remark 2.2 and Lemma 2.5, it is enough to know only those $z(G)$ of stable graphs $G \in \mathcal{G}$.

Remark 5.3. For convenience, we assume that the empty graph $\emptyset$ is the unique element in $\mathcal{G}(0)$ and $z(\emptyset)=1$. This is consistent with $a_{0}=1$.

First we recall Loi's recursion formula 22 .

$$
a_{k}(x)=-R_{k}(1)-\sum_{\substack{i+j=k \\ i, j \geq 1}} a_{i}(x) a_{j}(x)-\left.\sum_{\substack{\ell+i+j=k \\ 1 \leq \ell \leq k-1}} R_{\ell}\left(a_{i}(x, y) a_{j}(y, x)\right)\right|_{y=x} .
$$

It was pointed out to the author recently that essentially the same identity was also obtained independently in 10 .

Remark 5.4. Here we outline the strategy of our proof of the closed formulae for $z(G)$ in Theorem 1.2 , We will give a graph-theoretic interpretation of Loi's formula in Proposition 5.7 the key ingredient is the graphical formulae for derivatives of $\operatorname{det} g$ proved in Lemma 5.5. Then we extract the coefficient $z(G)$ for any given graph $G$ in Lemma 5.8 , which will be used to prove $z(G)=0$ for any connected but not strongly connected graph $G$ in Proposition 6.2. By specializing Lemma 5.8 to a strongly connected graph $G$, we prove in Lemma 5.9 that $z(G)$ can be written as a summation over equivalent classes of linear subgraphs of $G$, which implies the explicit closed formula in Proposition 6.7 through a classical result in spectral graph theory: the Coefficient Theorem. 
Next we introduce some notation. Let $\mathscr{L}$ be the set of digraphs consisting of a finite number of vertex-disjoint simple cycles (i.e. simple polygons without common vertex). The length of a simple cycle is defined to be the number of its edges. For each graph $L \in \mathscr{L}$, we can write $L$ as a finite increasing sequence of nonnegative integers $\left[i_{1}, \ldots, i_{m}\right]$, meaning $L$ consists of $m$ disjoint simple cycles, whose lengths are specified by $i_{1}, \ldots, i_{m}$. We define the index of $L$ to be

$$
i(L)=m+i_{1}+\cdots+i_{m} .
$$

Note that [0] is just a single vertex and [1] is a vertex with a self-loop. If $0 \notin L$, then $L$ is usually called a linear digraph. Recall that a linear directed graph is a digraph in which $\operatorname{deg}^{+}(v)=\operatorname{deg}^{-}(v)=1$ for each vertex $v$.

Given a set of indices $\alpha_{1}, \ldots, \alpha_{r}$, denote by $\mathscr{L}\left(\alpha_{1}, \ldots, \alpha_{r}\right)$ the isomorphism classes of all possible decorations of the vertices of $L \in \mathscr{L}$ with the half-edges $\alpha_{1}, \ldots, \alpha_{r}$ requiring each vertex to be semistable. Two decorations of $L$ that differ by a graph isomorphism are considered the same.

The following crucial lemma explains the graphical properties of the partial derivatives of $\operatorname{det} g$.

Lemma 5.5. We have

$$
\frac{1}{\operatorname{det} g} \partial_{\alpha_{1}} \ldots \partial_{\alpha_{r}} \operatorname{det} g=\sum_{\substack{L \in \mathscr{L}\left(\alpha_{1}, \ldots, \alpha_{r}\right) \\ 0 \notin L}}(-1)^{i(L)} \cdot L .
$$

Proof. By (17) and (18), we have

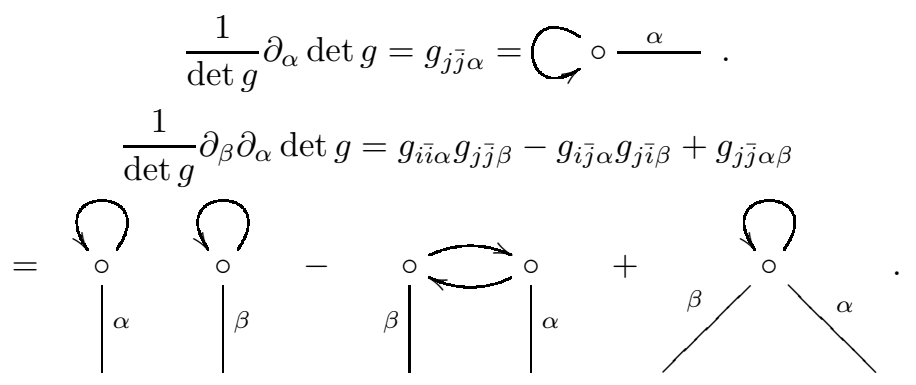

So equation (49) follows by an easy induction.

Definition 5.6. Let $L \in \mathscr{L}, G_{1}, G_{2} \in \mathcal{G}^{s s}$. We denote by $\mathscr{G}\left(L, G_{1}, G_{2}\right)$ the set of all different ways to add a finite number of directed edges to the disjoint union of three graphs $L, G_{1}, G_{2}$ satisfying that

i) No head of these new edges is connected to vertices of $G_{1}$ and no tail of these new edges is connected to vertices of $G_{2}$. Such $G_{1}$ and $G_{2}$ are called source and sink respectively.

ii) These new edges may act on edges of $G_{1}$ and $G_{2}$ as illustrated in Figure 4 .

iii) The final resulting digraph is semistable.

For $\mathcal{Z} \in \mathscr{G}\left(L, G_{1}, G_{2}\right)$, we denote by $[\mathcal{Z}]$ the resulted graph. Two configurations $\left[\mathcal{Z}_{1}\right],\left[\mathcal{Z}_{2}\right] \in$ $\mathscr{G}\left(L, G_{1}, G_{2}\right)$ are considered equal if there is an automorphism between $\left[\mathcal{Z}_{1}\right]$ and $\left[\mathcal{Z}_{2}\right]$ leaving $L$ invariant and keeping $G_{1}$ and $G_{2}$ fixed.

Given $k \geq 1$, we also define a function

$$
F_{k}\left(L, G_{1}, G_{2}\right)=\sum_{\substack{\mathcal{Z} \in \mathscr{G}\left(L, G_{1}, G_{2}\right) \\ \omega([\mathcal{Z}])=k}} \frac{(-1)^{i(L)+1}[\mathcal{Z}]}{|\operatorname{Aut}(\mathcal{Z})|},
$$

where $\operatorname{Aut}(\mathcal{Z})$ is the subgroup of the group of automorphism of $[\mathcal{Z}]$ leaving $L$ invariant and keeping $G_{1}$ and $G_{2}$ fixed. We may extend $F_{k}$ as a bilinear function in the second and third parameters.

Proposition 5.7. Given $k \geq 1$, we have

$$
a_{k}(x)=\sum_{\substack{L \in \mathscr{L}, G_{1}, G_{2} \in \mathcal{G}^{s s} \\ \omega\left(G_{1}\right)<k, \omega\left(G_{2}\right)<k}} z\left(G_{1}\right) z\left(G_{2}\right) F_{k}\left(L, G_{1}, G_{2}\right) .
$$

Note that we allow $L, G_{1}, G_{2}$ to be empty. 
Proof. In fact, (51) is just a graph-theoretic interpretation of Loi's formula (47)

$$
a_{k}(x)=-\left.\sum_{\substack{\ell+i+j=k \\ i<k, j<k}} R_{\ell}\left(a_{i}(x, y) a_{j}(y, x)\right)\right|_{y=x}
$$

We need to take a close look at the operators $R_{j}$ defined in (27). Note that the function $S$ in (27) plays the role of an isolated vertex $[0] \in \mathscr{L}$, the cycle with length zero. The linear subgraphs are contributed by derivatives of $\operatorname{det} g$ as described in Lemma 5.5. Moreover, $G_{1}$ and $G_{2}$ respectively represent $a_{j}(y, x)$ and $a_{i}(x, y)$ in the right-hand side of (52). The factorials in the denominator of (27) account for the removal of labels of the added edges and isolated vertices in each configuration. With each term of (27) thus interpreted, (51) follows readily from (52).

If $[\mathcal{Z}]=G$, we call $\mathcal{Z}$ a $G$-configuration (abbr. $G$-config). For a given digraph $G$, to specify a $G$-configuration is equivalent to specify three vertex-disjoint subgraphs $L, G_{1}, G_{2}$, where $L$ is a linear directed subgraph of $G$ without isolated vertex and $G_{1}, G_{2} \in \mathcal{G}^{s s}$ are respectively a source and a sink. Note that the union of $L, G_{1}, G_{2}$ may not contain all vertices of $G$. Let $\mathcal{L}$ be the set of all linear directed subgraphs $L$ of $G$. We may simply write $\mathcal{Z}=\left(L, G_{1}, G_{2}\right) \in G$-config and define a function

$$
F_{G}(\mathcal{Z})=\frac{(-1)^{i(\mathcal{Z})+1}}{|\operatorname{Aut}(\mathcal{Z})|}
$$

where $i(\mathcal{Z})$ is the index of $\mathcal{Z}$ defined by

$$
i(\mathcal{Z})=v(L)+p(L)+x(\mathcal{Z}) .
$$

Here $v(L)$ is the number of vertices in $L, p(L)$ is the number of components in $L$ and $x(\mathcal{Z})$ is the number of vertices of $G$ not belong to $L, G_{1}, G_{2}$.

Lemma 5.8. For any $G \in \mathcal{G}^{\text {ss }}$, we have

$$
z(G)=\sum_{\mathcal{Z} \in G \text {-config }} z\left(G_{1}\right) z\left(G_{2}\right) F_{G}(\mathcal{Z})
$$

where $\mathcal{Z}=\left(L, G_{1}, G_{2}\right)$ runs over all isomorphism classes of $G$-configurations.

Proof. It follows from Proposition 5.7.

Now we treat the case that $G \in \mathcal{G}^{s s}(k)$ is strongly connected. In the right-hand side of (47), the graphs from the second term are not connected. The graphs from the last term are not strongly connected, since it contains either a source or a sink. We see that only the first term $-R_{k}(1)$ contributes to $z(G)$ and all $G$-configurations are of the form $(L, \emptyset, \emptyset)$. It is not difficult to see that the automorphism group of the $G$-configuration $(L, \emptyset, \emptyset)$, denoted by $\operatorname{Aut}_{\mathrm{G}}(L)$, is the subgroup of $\operatorname{Aut}(G)$ leaving $L$ invariant. Two $G$-configurations $\left(L_{1}, \emptyset, \emptyset\right)$ and $\left(L_{2}, \emptyset, \emptyset\right)$ are equivalent if and only if $L_{1}=h\left(L_{2}\right)$ for some $h \in \operatorname{Aut}(G)$. This defines an equivalence relation $\sim$ on $\mathcal{L}$. So Lemma 5.8 specializes to the following formula for strongly connected graphs.

Lemma 5.9. For any strongly connected graph $G \in \mathcal{G}^{\text {ss }}$, we have

$$
z(G)=\sum_{L \in \mathcal{L} / \sim} \frac{(-1)^{|V|+1+p(L)}}{\left|\operatorname{Aut}_{G}(L)\right|},
$$

where $L$ runs over the equivalence classes of linear subgraphs of $G$ and $p(L)$ is the number of components in $L$. 


\section{Proof of Theorem 1.2}

Proposition 6.1. If $G \in \mathcal{G}^{\text {ss }}$ is not connected and we write $G$ as a disjoint union of connected subgraphs $G=G_{1} \cup \cdots \cup G_{m}$, then we have

$$
z(G)=\prod_{j=1}^{m} z\left(G_{j}\right) /\left|\operatorname{Sym}\left(G_{1}, \ldots, G_{m}\right)\right|,
$$

where $\operatorname{Sym}\left(G_{1}, \ldots, G_{m}\right)$ denote the permutation group of these $m$ connected subgraphs.

Proof. It follows from Lemma 4.5 .

By the above proposition, we may restrict to compute $z(G)$ for connected $G$. For a digraph $G=(V, E)$, we can partition $V$ into strongly connected components, namely the maximal strongly connected subgraphs of $G$. Among these strongly connected components, we have at least one sink (a component without outgoing edges) and one source (a component without incoming edges).

Proposition 6.2. Given a stable graph $G \in \mathcal{G}$ which is connected but not strongly connected, then we have $z(G)=0$.

Proof. By definition, any sink or source of a stable graph $G$ must be at least semistable. Without loss of generality, we may assume that $C \in \mathcal{G}^{s s}$ is a sink of $G$. We have a nonempty subgraph $G^{\prime}$ of $G$ containing all vertices not in $C$, such that there are $k$ arrows $e_{1}, \ldots, e_{k}$ from $G^{\prime}$ to $C$, as depicted in Figure 7.

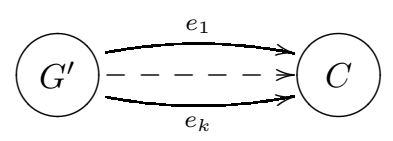

Figure 7. Decomposition of $G$ into a sink $C$ and $G^{\prime}$

In this case, any $G$-configuration is a union of $\mathcal{Z}^{\prime}=\left(L^{\prime}, G_{1}^{\prime}, G_{2}^{\prime}\right) \in G^{\prime}$-config and $\mathcal{Z}^{\prime \prime}=\left(L^{\prime \prime}, \emptyset, \emptyset\right) \in$ $C$-config. Since $G$ is stable, we can rule out Case (ii) of Definition 5.6 .

Let $\operatorname{Aut}_{f}(G)$ be the subgroup of $\operatorname{Aut}(G)$ fixing all edges $e_{1}, \ldots, e_{k}$. Let $\operatorname{Aut}_{f}(C)$ and $\operatorname{Aut}_{f}\left(\mathcal{Z}^{\prime \prime}\right)$ be respectively the subgroups of $\operatorname{Aut}(C)$ and $\operatorname{Aut}\left(\mathcal{Z}^{\prime \prime}\right)$ fixing the heads of the arrows $e_{i}, 1 \leq i \leq k$. We define $\operatorname{Aut}_{f}\left(\mathcal{Z}^{\prime}\right)$ to be the subgroup of $\operatorname{Aut}\left(\mathcal{Z}^{\prime}\right)$ fixing the tails of the arrows $e_{i}, 1 \leq i \leq k$.

Given a $G^{\prime}$-configuration $\mathcal{Z}^{\prime}=\left(L^{\prime}, G_{1}^{\prime}, G_{2}^{\prime}\right)$ and a $C$-configuration $\mathcal{Z}^{\prime \prime}=\left(L^{\prime \prime}, \emptyset\right.$, $\left.\emptyset\right)$, we define the following set

$$
S\left(\mathcal{Z}^{\prime}, \mathcal{Z}^{\prime \prime}\right)=\left\{\text { isomorphism classes in } G \text {-config of the form } \mathcal{Z}^{\prime} \coprod p\left(\mathcal{Z}^{\prime \prime}\right), p \in \frac{\operatorname{Aut}(C)}{\operatorname{Aut}_{f}(C)}\right\},
$$

where $p\left(\mathcal{Z}^{\prime \prime}\right)=\left(p\left(L^{\prime \prime}\right), \emptyset, \emptyset\right) \in C$-config and $\mathcal{Z}^{\prime} \coprod p\left(\mathcal{Z}^{\prime \prime}\right)=\left(L^{\prime} \coprod p\left(L^{\prime \prime}\right), G_{1}^{\prime}, G_{2}^{\prime}\right)$.

First we assume that all automorphisms of $G$ fix these $e_{i}, 1 \leq i \leq k$, namely $\operatorname{Aut}(G)=\operatorname{Aut}_{f}(G)$. By our assumption, for $\mathcal{Z}=\mathcal{Z}^{\prime} \coprod p\left(\mathcal{Z}^{\prime \prime}\right) \in S\left(\mathcal{Z}^{\prime}, \mathcal{Z}^{\prime \prime}\right)$ we have $\operatorname{Aut}(\mathcal{Z})=\operatorname{Aut}_{f}\left(\mathcal{Z}^{\prime}\right) \operatorname{Aut}_{f}\left(p\left(\mathcal{Z}^{\prime \prime}\right)\right)$

It is enough to show that for any fixed $G^{\prime}$-configuration $\mathcal{Z}^{\prime}=\left(L^{\prime}, G_{1}^{\prime}, G_{2}^{\prime}\right)$, the contribution to $z(G)$ from the $G$-configurations $\left(L^{\prime}, G_{1}^{\prime}, G_{2}^{\prime} \amalg C\right)$ and $\left\{S\left(\mathcal{Z}^{\prime}, \mathcal{Z}^{\prime \prime}\right)\right\}_{\mathcal{Z}^{\prime \prime} \in C \text {-config }}$ add up to zero.

We will proceed by induction on the weight of $G$. Namely we assume that if a semistable graph $H \in \mathcal{G}^{s s}$ is connected but not strongly connected and the weight of $H$ is less than the weight of $G$, then $z(H)=0$ (cf. Corollary 6.4).

First we assume $G_{2}^{\prime}=\emptyset$. By Lemma [5.8, the contribution to $z(G)$ from the $G$-configuration $\mathcal{Z}=\left(L^{\prime}, G_{1}^{\prime}, C\right)$ is

$$
\begin{aligned}
& z\left(G_{1}^{\prime}\right) z(c) \frac{(-1)^{i\left(\mathcal{Z}^{\prime}\right)+1}}{\left|\operatorname{Aut}_{f}\left(\mathcal{Z}^{\prime}\right)\right|} \times \frac{|\operatorname{Aut}(C)|}{\left|\operatorname{Aut}_{f}(C)\right|} \\
= & z\left(G_{1}^{\prime}\right) \frac{(-1)^{i\left(\mathcal{Z}^{\prime}\right)+1}}{\left|\operatorname{Aut}_{f}\left(\mathcal{Z}^{\prime}\right)\right|} \sum_{\mathcal{Z}^{\prime \prime} \in C \text {-config }} \frac{(-1)^{i\left(\mathcal{Z}^{\prime \prime}\right)+1}}{\left|\operatorname{Aut}\left(\mathcal{Z}^{\prime \prime}\right)\right|} \times \frac{|\operatorname{Aut}(C)|}{\left|\operatorname{Aut}_{f}(C)\right|} .
\end{aligned}
$$


The contribution to $z(G)$ from the set of $G$-configurations $\left\{S\left(\mathcal{Z}^{\prime}, \mathcal{Z}^{\prime \prime}\right)\right\}_{\mathcal{Z}^{\prime \prime} \in C \text {-config }}$ is

$$
z\left(G_{1}^{\prime}\right) \frac{1}{\left|\operatorname{Aut}_{f}\left(\mathcal{Z}^{\prime}\right)\right|} \sum_{\substack{\mathcal{Z}^{\prime \prime} \in C \text {-config } \\ \mathcal{Z}^{\prime} \amalg p\left(\mathcal{Z}^{\prime \prime}\right) \in S\left(\mathcal{Z}^{\prime}, \mathcal{Z}^{\prime \prime}\right)}} \frac{(-1)^{i\left(\mathcal{Z}^{\prime}\right)+i\left(\mathcal{Z}^{\prime \prime}\right)+1}}{\left|\operatorname{Aut}_{f}\left(p\left(\mathcal{Z}^{\prime \prime}\right)\right)\right|} .
$$

We now show that the contributions to $z(G)$ in (59) and (60) add up to zero, namely we need to prove that for any $\mathcal{Z}^{\prime \prime} \in C$-config

$$
\frac{1}{\left|\operatorname{Aut}\left(\mathcal{Z}^{\prime \prime}\right)\right|} \times \frac{|\operatorname{Aut}(C)|}{\left|\operatorname{Aut}_{f}(C)\right|}=\sum_{\mathcal{Z}^{\prime} \amalg p\left(\mathcal{Z}^{\prime \prime}\right) \in S\left(\mathcal{Z}^{\prime}, \mathcal{Z}^{\prime \prime}\right)} \frac{1}{\left|\operatorname{Aut}_{f}\left(p\left(\mathcal{Z}^{\prime \prime}\right)\right)\right|} .
$$

This can be seen as follows: Denote by $H$ the set of all possible distributions of the labeled heads of $e_{i}, 1 \leq i \leq k$ on $C$. Note that some of these $e_{i}$ may have the same head on $C$. It is obvious that $H$ is in one-to-one correspondence with $\operatorname{Aut}(C) / \operatorname{Aut}_{f}(C)$.

We have the natural action of $\operatorname{Aut}\left(\mathcal{Z}^{\prime \prime}\right)$ on $H$, then the set of orbits is just $S\left(\mathcal{Z}^{\prime}, \mathcal{Z}^{\prime \prime}\right)$ and the isotropy group at $\mathcal{Z}=\mathcal{Z}^{\prime} \coprod p\left(\mathcal{Z}^{\prime \prime}\right) \in S\left(\mathcal{Z}^{\prime}, \mathcal{Z}^{\prime \prime}\right)$ is just $\operatorname{Aut}_{f}\left(p\left(\mathcal{Z}^{\prime \prime}\right)\right)$.

If $G_{2}^{\prime} \neq \emptyset$ and $G_{2}^{\prime}$ is connected with a tail of some $e_{i}$, then the contribution to $z(G)$ from the $G$-configurations $\left(L^{\prime}, G_{1}^{\prime}, G_{2}^{\prime}+C\right)$ is zero by induction and all configurations in $\left\{S\left(\mathcal{Z}^{\prime}, \mathcal{Z}^{\prime \prime}\right)\right\} \mathcal{Z}^{\prime \prime} \in C$-config are not allowed. So we may assume that there are no edges between $G_{2}^{\prime}$ and $C$, then we have the same cancellation by Proposition 6.1. Note that $G_{2}^{\prime}+C$ denote the induced subgraph whose vertices are the union of vertices of $G_{2}^{\prime}$ and $C$.

If $\operatorname{Aut}(G) \neq \operatorname{Aut}_{f}(G)$, the above argument still works with appropriate modifications.

Remark 6.3. We will give an alternative proof of Proposition 6.2 in 38 . As pointed out by the referee, Proposition 6.2 should also follow from the path integral formula by Douglas and Klevtsov [14.

Corollary 6.4. If $G \in \mathcal{G}^{\text {ss }}$ is connected but not strongly connected, then $z(G)=0$.

Proof. Given any stable graph $H \in \mathcal{G}$, if we apply Lemma 2.5 to each vertices of $H$, we get a sum of semistable graphs of the same weight. Moreover, it is not difficult to see that if $H$ is strongly connected, all these semistable graphs are also strongly connected. So we conclude the proof.

Example 6.5. Let us illustrate the above proof by considering the following semistalbe graph $G$ in Figure 8, which is only weakly connected. Let $C$ be its unique sink and $G^{\prime}$ be its unique source. Then

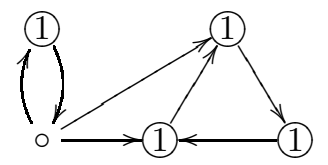

Figure 8. A semistable graph $G \in \mathcal{G}^{s s}(6)$

we have $|\operatorname{Aut}(C)|=3$ and $\left|\operatorname{Aut}_{f}(C)\right|=1$. For all $G$-configurations, we can check that the two sides of the equation (61) match, their values are listed in Table 2

TABLE 2. G-configurations

\begin{tabular}{|c|c|c|c|c|c|}
\hline$L^{\prime}$ & $\emptyset$ & {$[1]$} & {$\left[1^{2}\right]$} & {$\left[1^{3}\right]$} & {$[3]$} \\
\hline$\emptyset$ & 1 & 3 & 3 & 1 & 1 \\
\hline$[1]$ & 1 & 3 & 3 & 1 & 1 \\
\hline$[2]$ & 1 & 3 & 3 & 1 & 1 \\
\hline
\end{tabular}

The following theorem is called the coefficient theorem (see Theorem 1.2 in [1]) from the spectral graph theory. 
Theorem 6.6. Let $P_{G}(\lambda)=\lambda^{n}+c_{1} \lambda^{n-1}+\cdots+c_{n}$ be the characteristic polynomial of a digraph $G$ with $n$ vertices. Then for each $i=1, \ldots, n$,

$$
c_{i}=\sum_{L \in \mathcal{L}_{i}}(-1)^{p(L)}
$$

where $\mathcal{L}_{i}$ is the set of all linear directed subgraphs $L$ of $G$ with exactly $i$ vertices; $p(L)$ denotes the number of components of $L$.

Proposition 6.7. If $G=(V, E) \in \mathcal{G}^{s s}$ is strongly connected, then

$$
z(G)=(-1)^{|V|+1} \frac{\operatorname{det}(I-A)}{|\operatorname{Aut}(G)|} .
$$

Proof. Let $\mathcal{L}$ be the set of all linear directed subgraphs of $G$. We have the natural action of Aut $(G)$ on $\mathcal{L}$, given by $h \cdot L=h(L)$ for $h \in \operatorname{Aut}(G), L \in \mathcal{L}$. From our discussion at the end of Section 5 , the orbits of this action are just $\mathcal{L} / \sim$ and the isotropy group at $L \in \mathcal{L}$ is just $\operatorname{Aut}_{G}(L)$. By Theorem 6.6 and Lemma 5.9, we have

$$
\begin{aligned}
\operatorname{det}(I-A)=1+\sum_{i=1}^{n} c_{i} & =\sum_{L \in \mathcal{L} / \sim}(-1)^{p(L)}|\operatorname{Aut}(G) \cdot L| \\
& =|\operatorname{Aut}(G)| \sum_{L \in \mathcal{L} / \sim} \frac{(-1)^{p(L)}}{\left|\operatorname{Aut}_{G}(L)\right|} \\
& =|\operatorname{Aut}(G)|(-1)^{|V|+1} z(G),
\end{aligned}
$$

which is just the equation (62).

Corollary 6.8. If $G \in \mathcal{G}^{s s}$ is strongly connected, then $z(G)=0$ if and only if 1 is an eigenvalue of the characteristic polynomial of $G$.

Proof. It follows from Proposition 6.7.

Propositions 6.1, 6.2 and 6.7 together imply Theorem 1.2

Proposition 6.9. If $G=(k)$, the digraph with one vertex and $k \geq 2$ self-loops, we have

$$
z(G)=-\frac{k-1}{k !} .
$$

Proof. It follows from Lemma 4.3 or Proposition 6.7

Proposition 6.10. Let $G=m \underset{j}{i}$ (n) be a strongly connected stable graph with two vertices, namely $i j \neq 0$. Then we have

$$
z(G)= \begin{cases}\frac{i j-(1-m)(1-n)}{2 \cdot m ! n ! i ! j !} & \text { if } i=j \text { and } m=n \\ \frac{i j-(1-m)(1-n)}{m ! n ! i ! j !} & \text { otherwise. }\end{cases}
$$

Proof. The formula follows from Proposition 6.7.

Example 6.11. Fix a normal coordinate around $x \in M$. By Proposition 6.9, we get the well known

$$
a_{1}=-\frac{1}{2}[2]=-\frac{1}{2} g_{i \bar{i} \bar{j} \bar{j}}=\frac{1}{2} R_{i \bar{i} j \bar{j}}=\frac{1}{2} \rho,
$$

where $(i, \bar{i}),(j, \bar{j})$ are paired indices to be contracted.

By Proposition 6.9, Proposition 6.10 and Proposition 6.1, we have

$$
a_{2}=-\frac{1}{3}[(3)]+\frac{1}{2}[(1) \underset{1}{\stackrel{1}{\longrightarrow}}]+\frac{3}{8}[\stackrel{2}{\underset{2}{\rightleftharpoons}}]+\frac{1}{8}[(2) \mid(2)] \text {. }
$$


Written in terms of derivatives of the Kähler metric $g_{i \bar{j}}$, we get

$$
a_{2}=-\frac{1}{3} g_{i \bar{i} j \bar{j} k \bar{k}}+\frac{1}{2} g_{i \bar{i} k \bar{l}} g_{j \bar{j} l \bar{k}}+\frac{3}{8} g_{i \bar{j} k \bar{l}} g_{j \bar{i} l \bar{k}}+\frac{1}{8} g_{i \bar{i} j \bar{j}} g_{k \bar{k} l \bar{l}} .
$$

It is understood that $(i, \bar{i}),(j, \bar{j}),(k, \bar{k}),(l, \bar{l})$ are paired indices to be contracted.

Apply the operator $D$ (defined in Remark 2.2) to the right-hand side of (67) and use the identities

$$
\begin{gathered}
D\left(g_{i \bar{j} k \bar{l}}\right)=-R_{i \bar{j} k \bar{l}}, \\
D\left(g_{i \bar{i} j \bar{j} k \bar{k}}\right)=-R_{i \bar{i} j \bar{j} ; k \bar{k}}+R_{k \bar{s} s \bar{j}} R_{i \bar{k} j \bar{s}}+R_{j \bar{j} s \bar{i}} R_{k \bar{k} i \bar{s}}+R_{i \bar{i} s \bar{j}} R_{k \bar{k} j \bar{s}} \\
=-\Delta \rho+|R|^{2}+2|R i c|^{2},
\end{gathered}
$$

we arrived at

$$
a_{2}=\frac{1}{3} \Delta \rho+\frac{1}{24}|R|^{2}-\frac{1}{6}|R i c|^{2}+\frac{1}{8} \rho^{2},
$$

which is the same as the function $a_{2}(x)$ computed in [23. We computed $a_{3}$ in Appendix $\mathrm{A}$ and we can use tables at Appendix B to compute $a_{4}$.

\section{Computations of $z(G)$}

In this section, we derive some explicit formulae of $z(G)$.
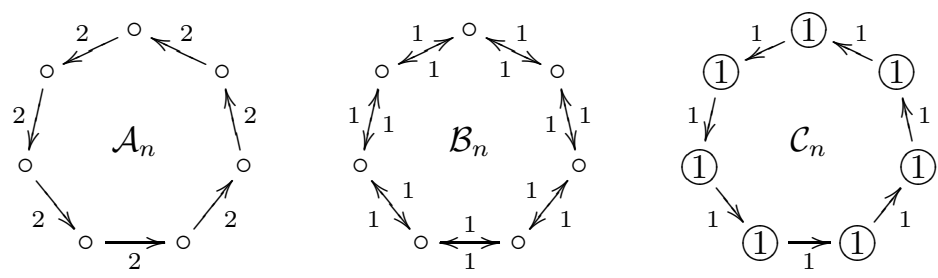

Figure 9. Three types of stable graphs in $\mathcal{G}_{n, 2 n}$

Proposition 7.1. Given $n \geq 3$, for the three graphs in Figure 9, we have

$$
\begin{aligned}
z\left(\mathcal{A}_{n}\right) & =\frac{(-1)^{n}\left(2^{n}-1\right)}{2^{n} n}, \\
z\left(\mathcal{B}_{n}\right) & =\frac{(-1)^{n}}{2 n}+\sum_{i=0}^{[n / 2]}(-1)^{n+i+1} \sum_{H \in \mathscr{C}_{i, n-2 i}} \frac{1}{\operatorname{Aut}(H)} \\
& =\left\{\begin{array}{ll}
0, & n \equiv 0 \\
(-1)^{n} /(2 n), & n \equiv \pm 1 \\
3(-1)^{n} /(2 n), & n \equiv \pm 2 \\
2(-1)^{n} / n, & n \equiv 3
\end{array} \quad \bmod 6,\right. \\
z\left(\mathcal{C}_{n}\right) & =\frac{(-1)^{n}}{n} .
\end{aligned}
$$

Here $\mathscr{C}_{i, j}$ denote the set of 2 -colored undirected cycle graphs with $i$ black vertices and $j$ white vertices and $\operatorname{Aut}(H)$ is the group of color-preserving automorphisms. Note the first equation for $z\left(\mathcal{B}_{n}\right)$ holds only when $n \geq 5$. 
Proof. Now we will compute $z(G)$ for $G=\mathcal{A}_{n}, \mathcal{B}_{n}, \mathcal{C}_{n}$. Since these graphs are strongly connected, all $G$-configurations are simply linear subgraphs $L$ of $G$. Two linear subgraphs $L_{1}$ and $L_{2}$ define the same $G$-configuration if there is an automorphism $\varphi$ of $G$ such that $\varphi\left(L_{1}\right)=L_{2}$.

i) When $G=\mathcal{A}_{n}$, it is easy to see that there are only two $\mathcal{A}_{n}$-configurations given by empty graph $\emptyset$ and a $n$-cycle $[n]$, respectively. By (56), we have

$$
\begin{aligned}
z\left(\mathcal{A}_{n}\right)=\frac{(-1)^{n+1}}{\left|\operatorname{Aut}\left(\mathcal{A}_{n}\right)\right|}+\frac{(-1)^{n}}{\left|\operatorname{Aut}_{\mathcal{A}_{n}}([n])\right|} & =\frac{(-1)^{n+1}}{2^{n} n}+\frac{(-1)^{n}}{n} \\
& =\frac{(-1)^{n}\left(2^{n}-1\right)}{2^{n} n} .
\end{aligned}
$$

ii) When $G=\mathcal{B}_{n}$, the contribution to $z\left(\mathcal{B}_{n}\right)$ by empty graph is

$$
\frac{(-1)^{n+1}}{\operatorname{Aut}\left(\mathcal{B}_{n}\right)}=\frac{(-1)^{n+1}}{2 n}
$$

where $\operatorname{Aut}\left(\mathcal{B}_{n}\right)$ is the dihedral group.

The contribution to $z\left(\mathcal{B}_{n}\right)$ by a $n$-cycle $[n]$ is

$$
\frac{(-1)^{n}}{\left|\operatorname{Aut}_{\mathcal{B}_{n}}([n])\right|}=\frac{(-1)^{n}}{n}
$$

All other $\mathcal{B}_{n}$-configurations are given by disjoint cycles of length two

$$
\left[2^{i}\right], \quad 0 \leq i \leq[n / 2],
$$

which are in one-to-one correspondence with the set $\mathscr{C}_{i, n-2 i}$ consisting of 2-colored undirected cycle graphs with $i$ black vertices and $n-2 i$ white vertices. Their contributions to $z\left(\mathcal{B}_{n}\right)$ are equal to

$$
(-1)^{n+i+1} \sum_{H \in \mathscr{C}_{i, n-2 i}} \frac{1}{\operatorname{Aut}(H)}
$$

where $\operatorname{Aut}(H)$ is the group of color-preserving automorphism. Summing up, we get the first equation for $z\left(\mathcal{B}_{n}\right)$. The second equation follows from a computation of $\operatorname{det}\left(I-A\left(\mathcal{B}_{n}\right)\right)$.

iii) When $G=\mathcal{C}_{n}$, we use $\operatorname{det}\left(I-A\left(\mathcal{C}_{n}\right)\right)=-1$.

Proposition 7.2. Let $n, m \geq 2$.

i) Let $K_{n}$ be the complete digraph with $n$ vertices and every pair of vertices is an edge, including a loop at each vertex. Namely every entry of the adjacency matrix of $K_{n}$ is 1 . Then

$$
z\left(K_{n}\right)=(-1)^{n}(n-1) / n !
$$

ii) Let $D_{n}$ be the de Bruijn graph of degree $n$. The graph $D_{n}$ has $2^{n-1}$ vertices, which are the sequences of 0 's and 1 's with length $n-1$. There is an edge from $a_{1} a_{2} \ldots a_{n-1}$ to $b_{1} b_{2} \ldots b_{n-1}$ if and only if $a_{2} a_{3} \ldots a_{n-1}=b_{1} b_{2} \ldots b_{n-2}$. Then

$$
z\left(D_{n}\right)=1 / 2 .
$$

iii) Let $K_{m, n}\left(V_{1}, V_{2}\right)$ be the complete bipartite digraph with $\left|V_{1}\right|=m,\left|V_{2}\right|=n$. Every vertex in $V_{1}$ has an arrow to every vertex in $V_{2}$ and vice versa. Then

$$
z\left(K_{m, n}\right)=\frac{m n-1}{\left(1+\delta_{m, n}\right) m ! n !},
$$

where $\delta_{m, n}=0$ if $m \neq n$ and $\delta_{n, n}=1$.

Proof. First note that $K_{n} \in \mathcal{G}\left(n^{2}-n\right), D_{n} \in \mathcal{G}\left(2^{n-1}\right)$ and $K_{m, n} \in \mathcal{G}(2 m n-m-n)$ all are strongly connected digraphs. It is well-known that their characteristic polynomials are respectively given by

$$
\begin{gathered}
\operatorname{det}\left(\lambda I-A\left(K_{n}\right)\right)=\lambda^{n-1}(\lambda-n), \\
\operatorname{det}\left(\lambda I-A\left(D_{n}\right)\right)=\lambda^{2^{n-1}-1}(\lambda-2), \\
\operatorname{det}\left(\lambda I-A\left(K_{m, n}\right)\right)=(-1)^{m+n} \lambda^{m+n-2}\left(\lambda^{2}-m n\right) .
\end{gathered}
$$


We also have $\left|\operatorname{Aut}\left(K_{n}\right)\right|=n !,\left|\operatorname{Aut}\left(D_{n}\right)\right|=2$ and $\left|\operatorname{Aut}\left(K_{m, n}\right)\right|=\left(1+\delta_{m, n}\right) m ! n !$, we conclude the proof by Proposition 6.7 .

Remark 7.3. The computation of $z(G)$ is reduced to study the spectra and the automorphism group of $G$. A detailed study of the spectral properties of graphs can be found at [1], which also contains a chapter on the relations between spectra of a graph and its automorphism group.

Let $U$ be the unit ball of $\mathbb{C}^{N}$ and $g_{i \bar{j}}$ is the Bergman metric on it,

$$
g_{i \bar{j}}(z)=\frac{\left(1-|z|^{2}\right) \delta_{i j}+\bar{z}_{i} z_{j}}{\left(1-|z|^{2}\right)^{2}}
$$

we know that $U$ is a normal coordinate with center 0 for $g_{i \bar{j}}$ and the curvature is constant

$$
R_{i \bar{j} k \bar{l}}=-\left(g_{i \bar{j}} g_{k \bar{l}}+g_{i \bar{l}} g_{k \bar{j}}\right) .
$$

The weighted reproducing kernel for $\left(U, g_{i \bar{j}}\right)$ is

$$
K_{\beta}(z)=\frac{\Gamma(\beta)}{\pi^{N} \Gamma(\beta-N)}\left(1-|z|^{2}\right)^{-\beta} .
$$

We know that $K_{\beta}(z)$ has an asymptotic expansion [3, 15]

$$
K_{\beta}(z)=(\beta / \pi)^{N} e^{\beta \Phi(z)} \sum_{k=0}^{\infty} a_{k}(z) \beta^{-k},
$$

where $a_{k}(z)$ is the $k$-th coefficient of Tian-Yau-Zelditch expansion and the potential $\Phi(z)$ is given by $\Phi(z)=-\log \left(1-|z|^{2}\right)$.

So in the case of unit ball equipped with the Bergman metric, $a_{k}$ equals the polynomial

$$
P_{k}=\sum_{1 \leq i_{1}<i_{2}<\cdots<i_{k} \leq N}\left(-i_{1}\right) \cdots\left(-i_{k}\right) .
$$

In particular,

$$
P_{1}=-\frac{N^{2}}{2}-\frac{N}{2}, \quad P_{2}=\frac{N^{4}}{8}+\frac{N^{3}}{12}-\frac{N^{2}}{8}-\frac{N}{12} .
$$

Lemma 7.4. Let $g_{i \bar{j}}$ be the Bergman metric on the unit ball $U$ of $\mathbb{C}^{N}$. Then $g_{i \bar{j} \alpha_{1} \alpha_{2} \ldots \alpha_{r}}(0)$ is nonzero only if the number of barred and unbarred indices in $\left\{\alpha_{1}, \alpha_{2}, \ldots, \alpha_{r}\right\}$ are equal. In this case, we have

$$
g_{i_{1} \bar{j}_{1} i_{2} \bar{j}_{2} \ldots i_{k} \bar{j}_{k}}(0)=(k-1) ! \sum_{\sigma \in S_{k}} g_{i_{1} \bar{j}_{\sigma(1)}} g_{i_{2} \bar{j}_{\sigma(2)}} \ldots g_{i_{k} \bar{j}_{\sigma(k)}}(0) \text {. }
$$

Proof. The first assertion can be proved by an induction. Let us prove (77). By (11) and (173), on $U$ we have

$$
g_{i_{1} \bar{j}_{1} i_{2} \bar{j}_{2}}=g_{i_{1} \bar{j}_{1}} g_{i_{2} \bar{j}_{2}}+g_{i_{1} \bar{j}_{2}} g_{i_{2} \bar{j}_{1}}+g^{m \bar{n}} g_{m \bar{j}_{1} \bar{j}_{2}} g_{i_{1} \bar{n} i_{2}} \text {. }
$$

As we have discussed in Section 2, both sides of the above equation may be represented by trees with half-edges and taking partial derivatives may be regarded as the action of half-edges on trees.

Let us look at the coefficients of $g_{i_{1} \bar{j}_{1}} g_{i_{2} \bar{j}_{2}} \ldots g_{i_{k} \bar{j}_{k}}$ after taking partial derivatives $\partial_{i_{3} \bar{j}_{3} \ldots i_{k} \bar{j}_{k}}$ to the right-hand side of (78). In fact, all contributions come from taking paired derivatives $\partial_{i_{3}} \bar{j}_{3} \ldots \partial_{i_{k}} \bar{j}_{k}$ consecutively to the first term in the right-hand side of (78). The coefficient is easily seen to be $2 \cdot 3 \cdots(k-1)=(k-1) !$

A cycle decomposition of a digraph $G$ is a partition of the edges of $G$ into edge-disjoint cycles (i.e. closed directed paths having no common edge). Let $\mathscr{C}_{G}$ denote the set of all cycle decomposition of $G$. It is well known that $G$ admits a cycle decomposition if and only if it $\operatorname{deg}^{+}(v)=\operatorname{deg}^{-}(v)$ for each vertex $v$ of $G$. 
Proposition 7.5. Given $k \geq 1$, we have

$$
\sum_{G \in \mathcal{G}(k)}(-)^{n(G)} \frac{\operatorname{det}(A-I)}{|\operatorname{Aut}(G)|} \prod_{v \in V}\left(\operatorname{deg}^{+}(v)-1\right) ! \sum_{H \in \mathscr{C}_{G}} N^{p(H)}=P_{k}
$$

where $v$ runs over all vertices of $G, n(G)$ is the number of components of $G$ and $p(H)$ is the number of cycles in the cycle decomposition $H \in \mathscr{C}_{G}$. The graph $G$ in the left-hand side need only run over all balanced graphs.

Proof. From $a_{k}=P_{k}$ and Lemma 7.4, we have

$$
\sum_{G \in \mathcal{G}(k)} z(G) \prod_{v \in V}\left(\operatorname{deg}^{+}(v)-1\right) ! \sum_{H \in \mathscr{C}_{G}} N^{p(H)}=P_{k} .
$$

Note that if $G \in \mathcal{G}$ is a disjoint union of connected subgraphs $G=G_{1} \cup \cdots \cup G_{n}$, then by Theorem 1.2 we have

$$
z(G)= \begin{cases}\frac{(-1)^{n} \operatorname{det}(A-I)}{|\operatorname{Aut}(G)|}, & \text { if all } G_{i} \text { are strongly connected; } \\ 0, & \text { otherwise. }\end{cases}
$$

So we get the desired formula.

Corollary 7.6. The leading term of the polynomial $P_{k}$ is $\frac{(-1)^{k}}{2^{k} k !} N^{2 k}$.

Proof. It follows from Proposition 6.1 and Proposition 6.9.

Let $G$ be a digraph. An Euler tour is a directed closed path in $G$ which visits each edge exactly once. As a generalization of the "Seven Bridges of Königsberg" problem, Euler showed that $G$ has an Euler tour if and only if $G$ is connected and $\operatorname{deg}^{+}(v)=\operatorname{deg}^{-}(v)$ at every vertex $v$. We denote by $\epsilon(G)$ the number of Euler tours in $G$ starting with a fixed edge.

Corollary 7.7. Given $k \geq 1$, we have

$$
\sum_{G \in \mathcal{G}(k)} z(G) \cdot \epsilon(G) \cdot \prod_{v \in V}\left(\operatorname{deg}^{+}(v)-1\right) !=\frac{(-1)^{k+1}}{k} B_{k},
$$

where $B_{k}$ is the $k$-th Bernoulli number: $B_{0}=1, B_{1}=-1 / 2, B_{2}=1 / 6, B_{3}=0$.

Proof. Using Barnes' asymptotic formula for $\Gamma$ functions, we have the following equality of power series (cf. [15])

$$
\sum_{k=0}^{\infty} P_{k} x^{k}=\exp \left(q_{1} x+q_{2} x^{2}+\ldots\right)
$$

where $q_{1}, q_{2}, \ldots$ are polynomials in $N$,

$$
q_{j}=\frac{1}{j(j+1)} \sum_{i=0}^{j}(-1)^{i+1}\left(\begin{array}{c}
j+1 \\
i
\end{array}\right) B_{i} N^{j+1-i} .
$$

Note the left-hand side of (82) is the coefficient of $N$ in $a_{k}$, which is equal to the coefficient of $N$ in $q_{k}$. The latter is just $\frac{(-1)^{k+1}}{k} B_{k}$.

It is interesting to note that the factor $\prod_{v \in V}\left(\operatorname{deg}^{+}(v)-1\right)$ ! also appears in the formula (cf. 34, p.56])

$$
\epsilon(G)=\tau(G) \prod_{v \in V}\left(\operatorname{deg}^{+}(v)-1\right) !
$$

where $\tau(G)$ is the number of oriented spanning subtrees of $G$ with a fixed root. An oriented tree $T$ with a root $v$ means that the underlying undirected graph is a tree and all arrows in $T$ point toward $v$. 
Let us check (82). When $k=1$ and $k=2$, by (65) and (66), the left-hand side of (82) respectively equals to

$$
\begin{gathered}
-\frac{1}{2} \cdot 1 \cdot 1=-\frac{1}{2}=B_{1}, \\
-\frac{1}{3} \cdot 2 \cdot 2+\frac{1}{2} \cdot 1 \cdot 1+\frac{3}{8} \cdot 2 \cdot 1=-\frac{1}{12}=\frac{-B_{2}}{2} .
\end{gathered}
$$

When $k=3$, by (87) in Appendix A, we have

$$
\begin{gathered}
z_{4} \cdot 1 \cdot 1+z_{5} \cdot 2 \cdot 1+z_{6} \cdot 1 \cdot 1+z_{7} \cdot 3 \cdot 1+z_{9} \cdot 2 \cdot 2 \\
+z_{10} \cdot 4 \cdot 2+z_{14} \cdot 6 \cdot 6+z_{15} \cdot 4 \cdot 1=0=\frac{B_{3}}{3} .
\end{gathered}
$$

\section{Appendix A. Computations of $a_{3}$}

We will express $a_{3}$ in terms of the following basis as used by Engliš [15].

$$
\begin{gathered}
\sigma_{1}=\rho^{3}, \quad \sigma_{2}=\rho R_{i \bar{j}} R_{j \bar{i}}, \quad \sigma_{3}=\rho R_{i \bar{j} k \bar{l}} R_{j \bar{i} \bar{l} \bar{k}}, \\
\sigma_{4}=R_{i \bar{j}} R_{k \bar{l}} R_{j \bar{j} l \bar{k}}, \quad \sigma_{5}=R_{i \bar{j}} R_{k \bar{i} l \bar{m}} R_{j \bar{k} m \bar{l}}, \quad \sigma_{6}=R_{i \bar{j}} R_{j \bar{k}} R_{k \bar{i}}, \\
\sigma_{7}=R_{i \bar{j} k \bar{l}} R_{j \bar{j} m \bar{n}} R_{l \bar{k} n \bar{m}}, \quad \sigma_{8}=\rho \Delta \rho, \quad \sigma_{9}=R_{i \bar{j}} R_{j \bar{i} ; k \bar{k}}, \\
\sigma_{10}=R_{i \bar{j} k \bar{l}} R_{j \bar{i} l \bar{k} ; m \bar{m}}, \quad \sigma_{11}=\rho_{; i} \rho_{; \bar{i}}, \quad \sigma_{12}=R_{i \bar{j} ; k} R_{j \bar{j} ; \bar{k}}, \\
\sigma_{13}=R_{i \bar{j} k \bar{l} ; m} R_{j \bar{i} l \bar{k} ; \bar{m}}, \quad \sigma_{14}=\Delta^{2} \rho, \quad \sigma_{15}=R_{i \bar{j} k \bar{l}} R_{j \bar{m} l \bar{n}} R_{m \bar{i} n \bar{k}} .
\end{gathered}
$$

We will compute the coefficients $c_{i}, 1 \leq i \leq 15$, such that

$$
a_{3}=c_{1} \sigma_{1}+c_{2} \sigma_{2}+\cdots+c_{15} \sigma_{15} .
$$

There are 15 stable graphs of weight 3 in $\mathcal{G}(3)$.

$$
\begin{aligned}
& \tau_{1}=[(2)|(2)|(2)], \quad \tau_{2}=\left[(1) \underset{1}{2}(1) \mid(2), \quad \tau_{3}=[\stackrel{2}{\stackrel{2}{2}} \circ \mid(2)],\right.
\end{aligned}
$$

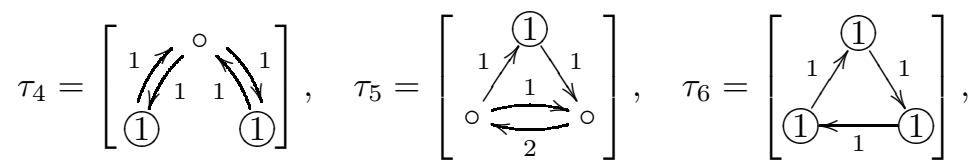

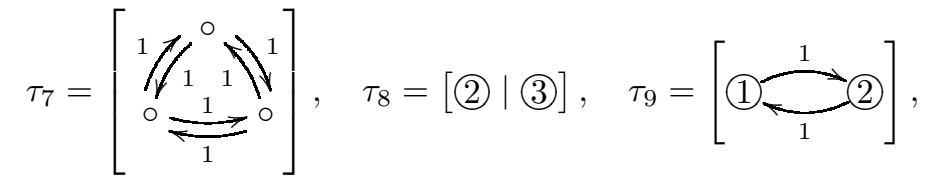

$$
\begin{aligned}
& \left.\tau_{10}=[1 \underset{2}{\stackrel{2}{\longrightarrow}} \cdot], \quad \tau_{11}=[(2) \stackrel{1}{\longrightarrow}(2)], \quad \tau_{12}=[1) \stackrel{1}{\underset{2}{\longrightarrow}(1)}\right] \text {, }
\end{aligned}
$$

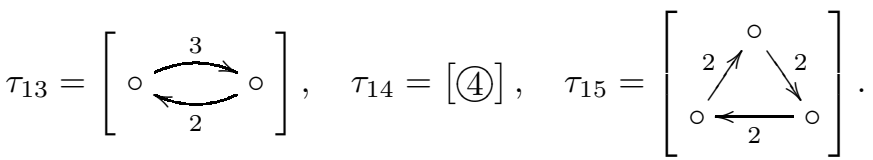

By Proposition 6.9, Proposition 6.10 and Proposition 6.1, we have

$$
a_{3}=\sum_{i=1}^{15} z\left(\tau_{i}\right) \tau_{i}=z_{1} \tau_{1}+z_{2} \tau_{2}+\cdots+z_{15} \tau_{15},
$$

where

$$
\begin{aligned}
z_{1}=-1 / 48, & z_{2}=-1 / 4, \quad z_{3}=-3 / 16, \quad z_{4}=0, \quad z_{5}=-1 \\
z_{6}=-1 / 3, \quad z_{7}=-2 / 3, \quad z_{8}=1 / 6, \quad z_{9}=1 / 2, & z_{10}=1
\end{aligned}
$$


A CLOSED FORMULA FOR THE ASYMPTOTIC EXPANSION OF THE BERGMAN KERNEL

$$
z_{11}=0, \quad z_{12}=1, \quad z_{13}=5 / 12, \quad z_{14}=-1 / 8, \quad z_{15}=-7 / 24
$$

We need to express each $\tau_{i}$ as a linear combination of $\sigma_{i}, 1 \leq i \leq 15$. By a standard computation as in Example 6.11, we get

$$
\begin{gathered}
\tau_{i}=-\sigma_{i}, \quad 1 \leq i \leq 7, \\
\tau_{8}=-2 \sigma_{2}-\sigma_{3}+\sigma_{8}, \quad \tau_{9}=-\sigma_{4}-\sigma_{5}-\sigma_{6}+\sigma_{9}, \quad \tau_{10}=-2 \sigma_{5}+\sigma_{10}-\sigma_{15}, \\
\tau_{11}=\sigma_{11}, \quad \tau_{12}=\sigma_{12}, \quad \tau_{13}=\sigma_{13}, \\
\tau_{14}=-3 \sigma_{4}-12 \sigma_{5}-3 \sigma_{6}+6 \sigma_{7}+7 \sigma_{9}+8 \sigma_{10}+10 \sigma_{12}+3 \sigma_{13}-\sigma_{14}-6 \sigma_{15}, \\
\tau_{15}=-\sigma_{15} .
\end{gathered}
$$

The only nontrivial computation is for $\tau_{14}$. By (11), we have

$$
\tau_{14}=g_{i \bar{i} j \bar{j} k \bar{k} l \bar{l}}=-\partial_{k \bar{k} l \bar{l}} R_{i \bar{i} j \bar{j}}+\partial_{k \bar{k} l \bar{l}}\left(g^{m \bar{n}} g_{m \bar{i} \bar{j}} g_{i \bar{n} j}\right) .
$$

So $\tau_{14}$ follows from a tedious but straightforward computation that

$$
\begin{aligned}
-\partial_{k \bar{k} l \bar{l}} R_{i \bar{i} j \bar{j}} & =-3 \sigma_{4}-6 \sigma_{5}-3 \sigma_{6}+6 \sigma_{7}+7 \sigma_{9}+4 \sigma_{10}+8 \sigma_{12}-\sigma_{14}-4 \sigma_{15}, \\
\partial_{k \bar{k} l \bar{l}}\left(g^{m \bar{n}} g_{m \bar{i} \bar{j}} g_{i \bar{n} j}\right) & =-6 \sigma_{5}+4 \sigma_{10}+2 \sigma_{12}+3 \sigma_{13}-2 \sigma_{15} .
\end{aligned}
$$

Substituting (88) into (86), we can get the coefficients in (85).

$$
\begin{gathered}
c_{1}=1 / 48, \quad c_{2}=-1 / 12, \quad c_{3}=1 / 48, \quad c_{4}=-1 / 8, \quad c_{5}=0, \\
c_{6}=5 / 24, \quad c_{7}=-1 / 12, \quad c_{8}=1 / 6, \quad c_{9}=-3 / 8, \quad c_{10}=0, \\
c_{11}=0, \quad c_{12}=-1 / 4, \quad c_{13}=1 / 24, \quad c_{14}=1 / 8, \quad c_{15}=1 / 24 .
\end{gathered}
$$

These results agree with the computations by Engliš [15]. Note that our convention of curvatures $R_{i \bar{j} k \bar{l}}, R_{i \bar{j}}, \rho$ in Section 2 all differ by a minus sign with that of $[15]$.

\section{Appendix B. TABles of $z(G)$ FOR $G \in \mathcal{G}(4)$}

By Proposition 6.1 and Corollary 6.4 we need only list $z(G)$ for the 51 strongly connected stable graphs in the following three tables. Note that if $G$ has $n$ vertices with the adjacency matrix $A$, then

$$
|\operatorname{Aut}(G)|=\prod_{1 \leq i, j \leq n} A_{i j} ! \cdot \#\left\{\varphi \in S_{n} \mid A_{\varphi(i), \varphi(j)}=A_{i j}, \forall 1 \leq i, j \leq n\right\}
$$

where $S_{n}$ is the symmetric group of $n$ elements.

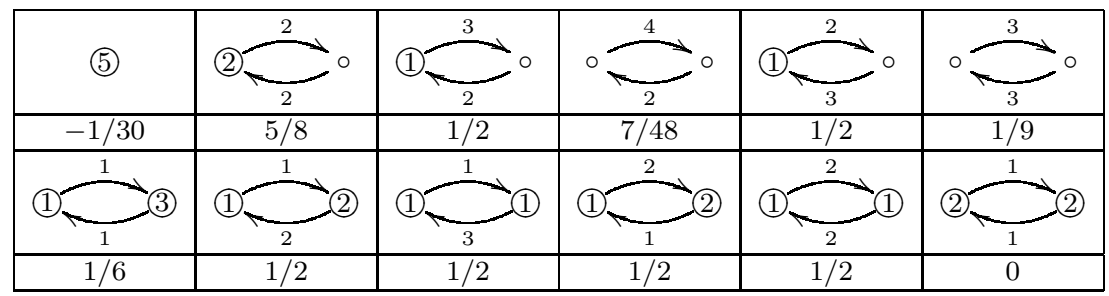

\section{REFERENCES}

[1] S. Alexakis, The decomposition of global conformal invariants I: On a conjecture of Deser and Schwimmer, arXiv:0711.1685

[2] R. Berman, B. Berndtsson and J. Sjöstrand, A direct approach to Bergman kernel asymptotics for positive line bundles, Ark. Mat. 46 (2008), 197-217.

[3] F. A. Berezin, Quantization, Math. USSR Izvest. 8 (1974), 1109-1163.

[4] D. Borthwick and A. Uribe, Nearly Kählerian embeddings of symplectic manifolds, Asian J. Math. 4 (2000) 599-620. 


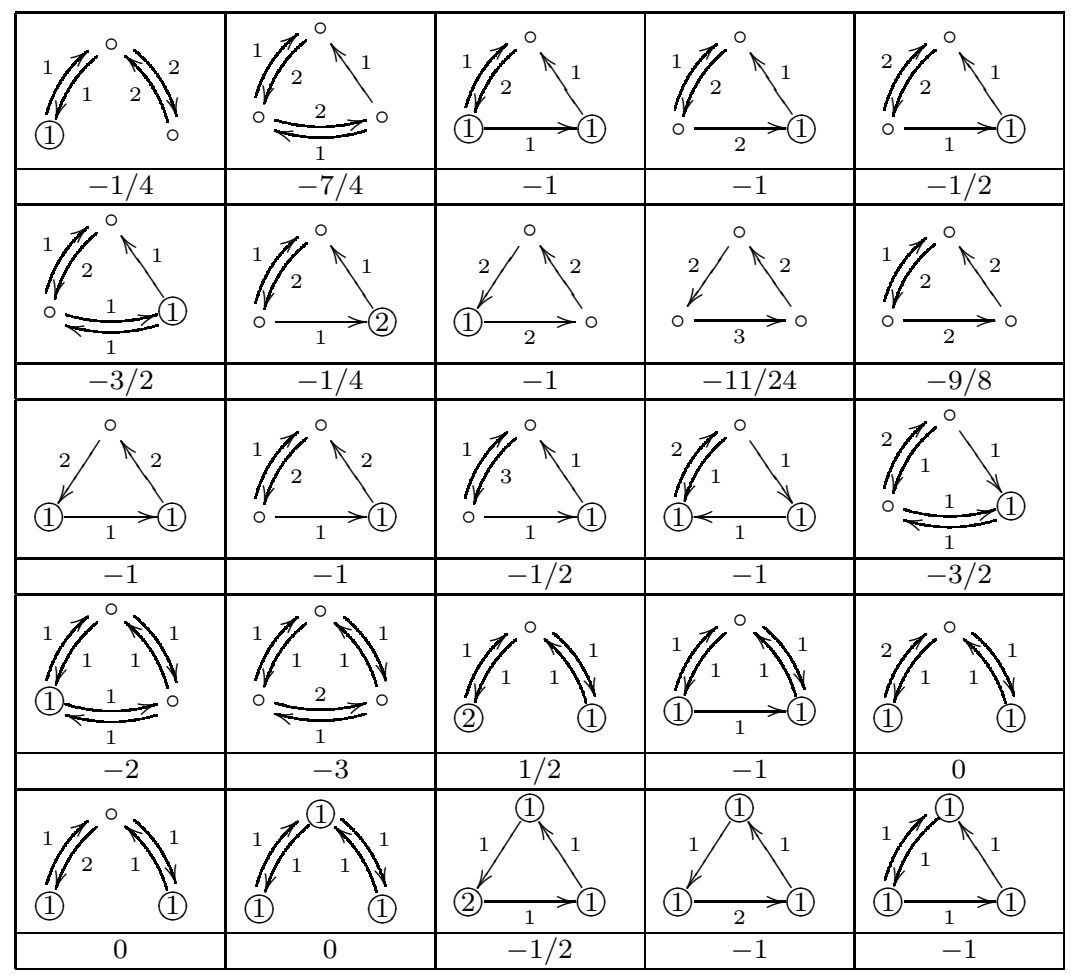

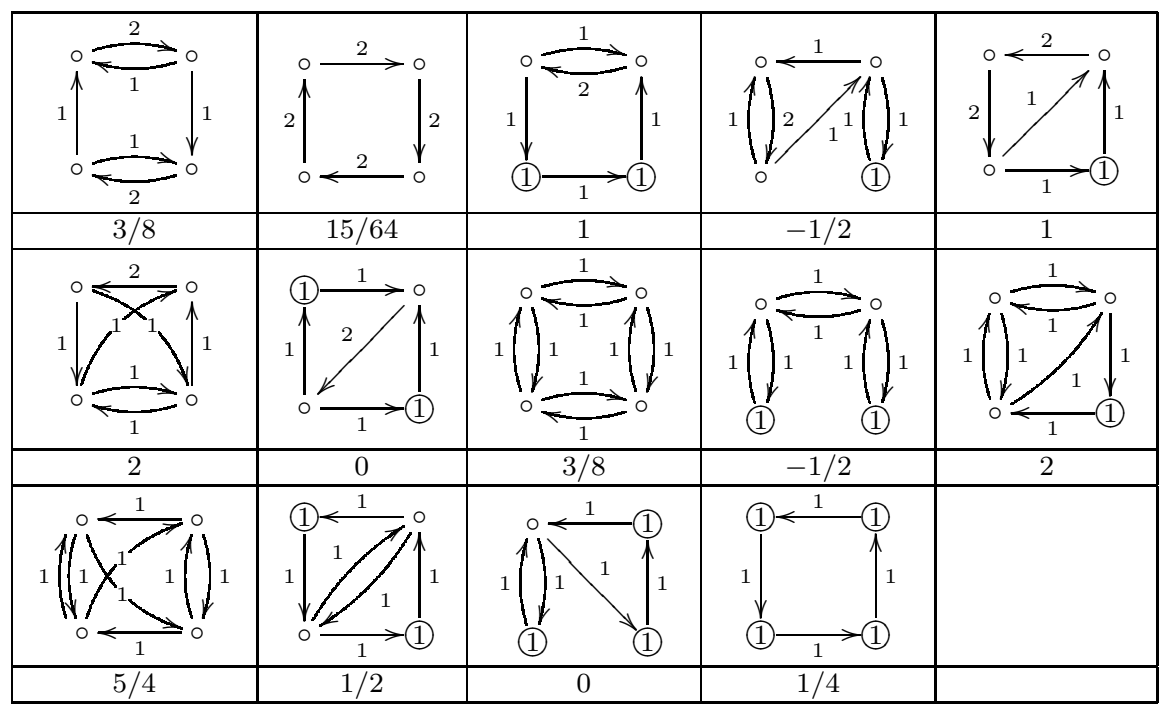

[5] T. Bouche, Convergence de la métrique de Fubini-Study d'un fibré linéaire positif, Ann Inst. Fourier 40 (1990), $117-130$.

[6] L. Boutet de Monvel and J. Sjöstrand, Sur la singularité des noyau de Bergman et de Szegö, Astérisque, 34-35 (1976), 123-164.

[7] M. Cahen, S. Gutt and J. Rawnsley, Quantization of Kähler manifolds II, Trans. Amer. Math. Soc. 337 (1993), 73-98.

[8] E. Calabi, Isometric imbeddings of complex manifolds, Ann. Math. 58 (1953), 1-23.

[9] D. Catlin, The Bergman kernel and a theorem of Tian, Analysis and geometry in several complex variables (Katata, 1997), 1999, 1-23.

[10] L. Charles, Berezin-Toeplitz operators, a semi-classical approach, Comm. Math. Phys. 239 (2003), 1-28.

[11] D. Cvetković, M. Doob and H. Sachs, Spectra of graphs, Academic Press, New York, 1980.

[12] X. Dai, K. Liu and X. Ma, On the asymptotic expansion of Bergman kernel, J. Differential Geom. 72 (2006), no. $1,1-41$. 
[13] S. Donaldson, Scalar curvature and projective embeddings, I, J. Differential Geom. 59 (2001), no. 3, 479-522.

[14] M. Douglas and S. Klevtsov, Bergman Kernel from Path Integral, Comm. Math. Phys. 293 (2010), 205-230.

[15] M. Engliš, The asymptotics of a Laplace integral on a Kähler manifold, J. Reine Angew. Math. 528 (2000), 1-39.

[16] M. Engliš, Weighted Bergman kernels and quantization, Comm. Math. Phys. 227 (2002), 211-241.

[17] C. Fefferman, Parabolic invariant theory in complex analysis, Adv. Math. 31 (1979), 131-262.

[18] K, Hirachi, Invariant theory of the Bergman kernel of strictly pseudoconvex domains, Sugaku Expositions $\mathbf{1 7}$ (2004), 151-169.

[19] A. Karabegov and M. Schlichenmaier, Identification of Berezin-Toeplitz deformation quantization, J. Reine Angew. Math. 540 (2001), 49-76.

[20] M. Kontsevich, Deformation quantization of Poisson manifolds, Lett. Math. Phys. 66 (2003), no. 3, $157-216$.

[21] C. Liu and Z. Lu, On the asymptotic expansion of Tian-Yau-Zelditch, arixv:1105.0221.

[22] A. Loi, The Tian-Yau-Zelditch asymptotic expansion for real analytic Kähler metrics, Int. J. Geom. Methods in Modern Phys. 1 (2004), 253-263.

[23] Z. Lu, On the lower order terms of the asymptotic expansion of Tian-Yau-Zelditch, Amer. J. Math. 122 (2000), no. 2, 235-273.

[24] Z. Lu and G. Tian, The log term of the Szegö kernel, Duke Math. J. 125 (2004), no. 2, 351-387.

[25] X. Ma and G. Marinescu, The first coefficients of the asymptotic expansion of the Bergman kernel of the spinc Dirac operator, Internat. J. Math. 17 (2006), no. 6, 737-759.

[26] X. Ma and G. Marinescu, Holomorphic Morse Inequalities and Bergman Kernels, Progress in Mathematics vol. 254, Birkhäuser Boston, Boston, MA (2007).

[27] X. Ma and G. Marinescu, Generalized Bergman kernels on symplectic manifolds, Adv. Math. 217 (2008), no. 4, $1756-1815$.

[28] X. Ma and G. Marinescu, Berezin-Toeplitz quantization of Kähler manifolds, J. Reine Angew. Math. 662 (2012), $1-56$.

[29] J. Ross and R. Thomas, Weighted Bergman kernels on orbifolds, arXiv:0907.5215.

[30] N. Reshetikhin and L. Takhtajan, Deformation quantization of Kähler manifolds, L.D. Faddeev's Seminar on Mathematical Physics, Amer. Math. Soc. Transl. Ser. 2, Vol. 201, Amer. Math. Soc., Providence, RI, 2000, 257276.

[31] W. Ruan, Canonical coordinates and Bergman metrics, Comm. Anal. Geom. 6 (1998), no. 3, 589-631.

[32] B. Shiffman and S. Zelditch, Asymptotics of almost holomorphic sections of ample line bundles on symplectic manifolds, J. Reine Angew. Math. 544 (2002), 181-222.

[33] J. Song, The Szegö kernel on an orbifold circle bundle, arXiv:math/0405071.

[34] R. Stanley, Enumerative combinatorics, volume 2, Cambridge University Press, 1999.

[35] G. Tian, On a set of polarized Kähler metrics on algebraic manifolds, J. Diff. Geom. 32 (1990), 99-130.

[36] L. Wang, Bergman kernel and stability of holomorphic vector bundles with sections, MIT Ph.D. Dissertation (2003), 85 pages.

[37] X. Wang, Canonical metrics on stable vector bundles, Comm. Anal. Geom. 13 (2005), no. 2, 253-285.

[38] H. Xu, An explicit formula for the Berezin star product, Lett. Math. Phys. (to appear).

[39] H. Xu, On a formula of Gammelgaard for Berezin-Toeplitz quantization, arXiv:1204.2259.

[40] S.-T. Yau, Nonlinear analysis in geometry, L'Énseignement Math. 33 (1987), 109-158.

[41] S. Zelditch, Szegö kernel and a theorem of Tian, Internat. Math. Res. Notices, 1998, no. 6, 317-331.

Department of Mathematics, Harvard University, Cambridge, MA 02138, USA

E-mail address: haoxu@math.harvard.edu 